\title{
The 13th Data Release of the Sloan Digital Sky Survey: First Spectroscopic Data from the SDSS-IV Survey Mapping Nearby Galaxies at Apache Point Observatory
}

Franco D. Albareti ${ }^{1,2,126}$, Carlos Allende Prieto ${ }^{3,4}$, Andres Almeida ${ }^{5}$, Friedrich Anders ${ }^{6}$, Scott Anderson ${ }^{7}$, Brett H. Andrews ${ }^{8}$ (1), Alfonso Aragón-Salamanca ${ }^{9}$ (1), Maria Argudo-Fernández ${ }^{10,11}$, Eric Armengaud ${ }^{12}$, Eric Aubourg ${ }^{13}$, Vladimir Avila-Reese ${ }^{14}$ (1), Carles Badenes $^{8}$ (10), Stephen Bailey ${ }^{15}$, Beatriz Barbuy ${ }^{16,17}$, Kat Barger $^{18}$ (1) Jorge Barrera-Ballesteros ${ }^{19}$ (1) , Curtis Bartosz ${ }^{7}$,

Sarbani Basu ${ }^{20}$ (1), Dominic Bates ${ }^{21}$, Giuseppina Battaglia ${ }^{3,4}$, Falk Baumgarten ${ }^{6,22}$, Julien Baur ${ }^{12}$, Julian Bautista ${ }^{23}$,

Timothy C. Beers ${ }^{24}$ (ㄷ), Francesco Belfiore ${ }^{25,26}$, Matthew Bershady ${ }^{27}$ (1), Sara Bertran de Lis ${ }^{3,4}$, Jonathan C. Bird ${ }^{28}$,

Dmitry Bizyaev 29,30,31 (10), Guillermo A. Blanc ${ }^{32,33,34}$, Michael Blanton ${ }^{35}$ (1), Michael Blomqvist ${ }^{36}$, Adam S. Bolton ${ }^{23,37}$,

J. Borissova ${ }^{38,39}$, Jo Bovy ${ }^{40,41}$ (1), William Nielsen Brandt ${ }^{42,43,44}$, Jonathan Brinkmann ${ }^{29}$, Joel R. Brownstein ${ }^{23}$ (1),

Kevin Bundy ${ }^{45}$ (1) , Etienne Burtin ${ }^{12}$, Nicolás G. Busca ${ }^{13}$, Hugo Orlando Camacho Chavez ${ }^{17,46}$, M. Cano Díaz ${ }^{14}$, Michele Cappellari $^{47}{ }^{(1)}$, Ricardo Carrera ${ }^{3,4}{ }^{(1)}$, Yanping Chen $^{48}$, Brian Cherinka ${ }^{19}$, Edmond Cheung ${ }^{45}$ (1) , Cristina Chiappini ${ }^{6}$, Drew Chojnowski $^{30}$ (10) Chia-Hsun Chuang $^{6}$, Haeun Chung ${ }^{49}$ (D) , Rafael Fernando Cirolini ${ }^{17,50}$, Nicolas Clerc ${ }^{51}$, Roger E. Cohen ${ }^{52}$, Julia M. Comerford ${ }^{53}$, Johan Comparat ${ }^{1,2}$, Janaina Correa do Nascimento ${ }^{17,54}$, Marie-Claude Cousinou ${ }^{55}$, Kevin Covey ${ }^{56}$ (1), Jeffrey D. Crane $e^{32}$ (10), Rupert Croft ${ }^{57}$, Katia Cunha ${ }^{58}$, Jeremy Darling ${ }^{53}$ (iD, James W. Davidson, Jr. ${ }^{59}$, Kyle Dawson ${ }^{23}$ (1), Luiz Da Costa $^{17,58}$, Gabriele Da Silva Ilha ${ }^{17,50}$, Alice Deconto Machado ${ }^{17,50}$, Timothée Delubac ${ }^{60}$, Nathan De Lee ${ }^{28,61}$, Axel De la Macorra ${ }^{14}$, Sylvain De la Torre ${ }^{36}$, Aleksandar M. Diamond-Stanic ${ }^{27}$, John Donor ${ }^{18}$, Juan Jose Downes ${ }^{14,62}$, Niv Drory ${ }^{63}$ (1), Cheng Du ${ }^{64}$, Hélion Du Mas des Bourboux ${ }^{12}$, Tom Dwelly ${ }^{51}$, Garrett Ebelke ${ }^{59}$, Arthur Eigenbrot ${ }^{27}$ (1), Daniel J. Eisenstein ${ }^{65}$, Yvonne P. Elsworth ${ }^{66,67}$, Eric Emsellem ${ }^{68,69}$ (1), Michael Eracleous ${ }^{42,43}$ (1), Stephanie Escoffier ${ }^{55}$, Michael L. Evans ${ }^{7}$, Jesús Falcón-Barroso ${ }^{3,4}$, Xiaohui Fan $^{70}{ }^{70}$, Ginevra Favole ${ }^{1,2}$, Emma Fernandez-Alvar $^{14}$, J. G. Fernandez-Trincado ${ }^{71}$, Diane Feuillet ${ }^{30}$ (1) , Scott W. Fleming ${ }^{72,73}$ (1) Andreu Font-Ribera ${ }^{15,45}$, Gordon Freischlad ${ }^{29,30}$,

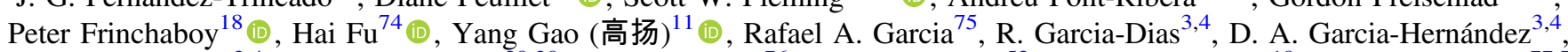
Ana E. Garcia Pérez $z^{3,4}$ (1) Patrick Gaulme ${ }^{29,30}$ (1) , Junqiang $\mathrm{Ge}^{76}$, Douglas Geisler ${ }^{52}$ (1) , Bruce Gillespie ${ }^{19}$, Hector Gil Marin 77,78 , Léo Girardi $^{17,79}$ (1D) Daniel Goddard ${ }^{80}$, Yilen Gomez Maqueo Chew ${ }^{14}$, Violeta Gonzalez-Perez ${ }^{80}$, Kathleen Grabowski ${ }^{29,30}$, Paul Green ${ }^{65}$ (10), Catherine J. Grier ${ }^{42,43}$ (1), Thomas Grier ${ }^{18,81}$, Hong Guo ${ }^{11}$ (i) , Julien Guy ${ }^{82}$, Alex Hagen ${ }^{42,43}$ (1) , Matt Hall ${ }^{59}$, Paul Harding ${ }^{83}$ (D), R. E. Harley ${ }^{56}$, Sten Hasselquist ${ }^{30}$, Suzanne Hawley $^{7}$ (D) Christian R. Hayes $^{59}$, Fred Hearty ${ }^{42}$, Saskia Hekker ${ }^{84}$ (D), Hector Hernandez Toledo ${ }^{14}$, Shirley Ho ${ }^{15,57}$, David W. Hogg ${ }^{35}$ (1), Kelly Holley-Bockelmann ${ }^{28}$ (1) , Jon A. Holtzman ${ }^{30}$ (10), Parker H. Holzer ${ }^{23}$, Jian Hu (胡剑 $)^{64,76}$ (1), Daniel Huber ${ }^{67,85,86}$ (1), Timothy Alan Hutchinson ${ }^{23}$ (1) , Ho Seong Hwang ${ }^{49}$ (1), Héctor J. Ibarra-Medel ${ }^{14}$, Inese I. Ivans ${ }^{23}$, KeShawn Ivory ${ }^{18,87}$, Kurt Jaehnig ${ }^{27}$, Trey W. Jensen ${ }^{23}$ (1), Jennifer A. Johnson ${ }^{88,89}$ (1), Amy Jones $^{90}$, Eric Jullo ${ }^{36}$ (1), T. Kallinger ${ }^{91}$ (1), Karen Kinemuchi ${ }^{29,30}{ }^{(0)}$, David Kirkby ${ }^{92}$, Mark Klaene ${ }^{29}$, Jean-Paul Kneib ${ }^{60}$,

Juna A. Kollmeier ${ }^{32}$ (10), Ivan Lacerna ${ }^{93}$ (1), Richard R. Lane ${ }^{93}$, Dustin Lang ${ }^{41}$ (D) , Pierre Laurent ${ }^{12}$, David R. Law ${ }^{72}$ (D),

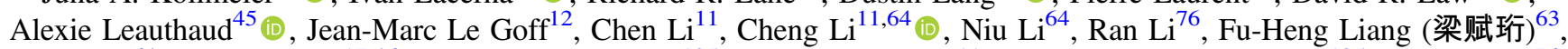

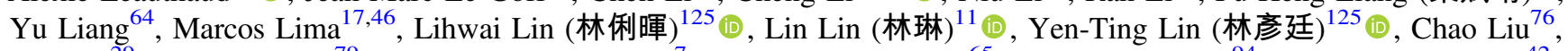
Dan Long $^{29}$, Sara Lucatello ${ }^{79}$ (1), Nicholas MacDonald ${ }^{7}$, Chelsea L. MacLeod ${ }^{65}$, J. Ted Mackereth ${ }^{94}$, Suvrath Mahadevan ${ }^{42}$ (1), Marcio Antonio Geimba Maia ${ }^{17,58}$, Roberto Maiolino ${ }^{25,26}$, Steven R. Majewski ${ }^{59}$ (1), Olena Malanushenko ${ }^{29,30}$, Viktor Malanushenko $^{29,30}$, Nícolas Dullius Mallmann ${ }^{17,54}$, Arturo Manchado ${ }^{3,4,95}$ (1) , Claudia Maraston $^{80}$,

Rui Marques-Chaves $^{3,4}\left(\mathbb{D}\right.$, Inma Martinez Valpuesta ${ }^{3,4}$, Karen L. Masters ${ }^{80}{ }^{(1)}$, Savita Mathur ${ }^{96}$, Ian D. McGreer ${ }^{70}$ (D), Andrea Merloni ${ }^{51}$, Michael R. Merrifield ${ }^{9}$ (10), Szabolcs Meszáros ${ }^{97}$, Andres Meza ${ }^{98}$ (1) Andrea Miglio ${ }^{66}$ (1) Ivan Minchev ${ }^{6}$ (1) Karan Molaverdikhani ${ }^{53,99}$, Antonio D. Montero-Dorta ${ }^{23}$, Benoit Mosser ${ }^{100}$, Demitri Muna ${ }^{88,89}$, Adam Myers ${ }^{101}$, Preethi Nair ${ }^{102}$,

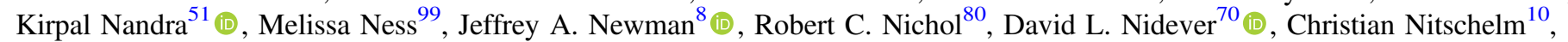
Julia O'Connell ${ }^{18}$ (10), Audrey Oravetz ${ }^{29,30}$, Daniel J. Oravetz ${ }^{29,30}$, Zachary Pace $^{27}$, Nelson Padilla ${ }^{93}$ (1),

Nathalie Palanque-Delabrouille ${ }^{12}$, Kaike Pan ${ }^{29,30}{ }^{(0)}$, John Parejko ${ }^{7}$, Isabelle Paris ${ }^{36}$, Changbom Park ${ }^{49}$, John A. Peacock ${ }^{103}$, Sebastien Peirani ${ }^{45,104}$, Marcos Pellejero-Ibanez ${ }^{3,4}$, Samantha Penny ${ }^{80}$, Will J. Percival ${ }^{80}$, Jeffrey W. Percival ${ }^{27}$, Ismael Perez-Fournon ${ }^{3,4}$ (1) Patrick Petitjean ${ }^{104}$, Matthew Pieri ${ }^{36}$, Marc H. Pinsonneault ${ }^{88}$ (1) , Alice Pisani ${ }^{55,104}$, Francisco Prada ${ }^{1,2,105}$, Abhishek Prakash ${ }^{8}$ (1), Natalie Price-Jones ${ }^{41}$, M. Jordan Raddick ${ }^{19}$, Mubdi Rahman ${ }^{19}$ (1), Anand Raichoor ${ }^{12}$, Sandro Barboza Rembold ${ }^{17,50}$, A. M. Reyna ${ }^{56}$, James Rich ${ }^{12}$, Hannah Richstein ${ }^{18}$, Jethro Ridl ${ }^{51}$, Rogemar A. Riffel ${ }^{17,50}$,

Rogério Riffel ${ }^{17,54}$, Hans-Walter Rix ${ }^{99}$ (i) , Annie C. Robin ${ }^{71}$, Constance M. Rockosi ${ }^{106}$, Sergio Rodríguez-Torres ${ }^{1}$,

Thaíse S. Rodrigues ${ }^{17,79,107}$, Natalie Roe ${ }^{15}$, A. Roman Lopes ${ }^{108}$, Carlos Román-Zúñiga ${ }^{109}$ (1), Ashley J. Ross ${ }^{89}$, Graziano Rossi ${ }^{110}$, John Ruan ${ }^{7}$ (1), Rossana Ruggeri ${ }^{80}$, Jessie C. Runnoe ${ }^{42,43}$, Salvador Salazar-Albornoz ${ }^{51}$, Mara Salvato ${ }^{51}$ (1),

Sebastian F. Sanchez ${ }^{14}$ (1), Ariel G. Sanchez ${ }^{51}$, José R. Sanchez-Gallego ${ }^{7}$, Basílio Xavier Santiago ${ }^{17,54}$, Ricardo Schiavon ${ }^{94}$, Jaderson S. Schimoia ${ }^{17,54}$ (1), Eddie Schlafly ${ }^{15,127}$ (1) , David J. Schlegel ${ }^{15}$ (D), Donald P. Schneider ${ }^{42,43}$, Ralph Schönrich ${ }^{47}$ (1) Mathias Schultheis ${ }^{111}$ (D) , Axel Schwope ${ }^{6}$, Hee-Jong Seo ${ }^{112}$, Aldo Serenelli ${ }^{113}$, Branimir Sesar ${ }^{99}$ (1) , Zhengyi Shao ${ }^{11}$, Matthew Shetrone $^{114}$ (1) , Michael Shull ${ }^{53}$ (1) , Victor Silva Aguirre ${ }^{67}$ (D) M. F. Skrutskie ${ }^{59}$, Anže Slosar ${ }^{115}$, Michael Smith ${ }^{27}$, Verne V. Smith ${ }^{116}$, Jennifer Sobeck ${ }^{59}$, Garrett Somers ${ }^{28,88}$, Diogo Souto ${ }^{58}$, David V. Stark ${ }^{45}$ (1) , Keivan G. Stassun ${ }^{28}$ (1), Matthias Steinmetz $^{6}$ (i), Dennis Stello ${ }^{85,117}$ (1), Thaisa Storchi Bergmann ${ }^{17,54}$, Michael A. Strauss ${ }^{18}$ (1), Alina Streblyanska ${ }^{3,4}$, 
Guy S. Stringfellow ${ }^{53}$ (10), Genaro Suarez ${ }^{109}$ (1) , Jing Sun ${ }^{18}$, Manuchehr Taghizadeh-Popp ${ }^{19}$, Baitian Tang ${ }^{52}$ (1) , Charling Tao $^{55,64}$, Jamie Tayar ${ }^{88}$ (10), Mita Tembe ${ }^{59}$, Daniel Thomas ${ }^{80}$, Jeremy Tinker $^{35}$, Rita Tojeiro ${ }^{21}$, Christy Tremonti ${ }^{27}$, Nicholas Troup ${ }^{59}$ (iD, Jonathan R. Trump ${ }^{42,127}$ (1), Eduardo Unda-Sanzana ${ }^{10}$, O. Valenzuela ${ }^{14}$, Remco Van den Bosch ${ }^{99}$ (1) , Mariana Vargas-Magaña ${ }^{119}$, Jose Alberto Vazquez ${ }^{115}$, Sandro Villanova ${ }^{52}$, M. Vivek $^{23}$, Nicole Vogt $^{30}$, David Wake ${ }^{120,121}$ (10), Rene Walterbos ${ }^{30}$ (i), Yuting Wang ${ }^{76,80}$, Enci Wang ${ }^{11}$ (10), Benjamin Alan Weaver ${ }^{35}$, Anne-Marie Weijmans ${ }^{21}$, David H. Weinberg ${ }^{88,89}$ (1), Kyle B. Westfall ${ }^{80}$, David G. Whelan ${ }^{122}$, Eric Wilcots ${ }^{27}$, Vivienne Wild ${ }^{21}$, Rob A. Williams ${ }^{94}$, John Wilson ${ }^{59}$, W. M. Wood-Vasey ${ }^{8}$ (1), Dominika Wylezalek ${ }^{19}$ (1), Ting Xiao (肖婷) ${ }^{11}$, Renbin Yan ${ }^{123}$ (1), Meng Yang ${ }^{64}$, Jason E. Ybarra ${ }^{109,124}$ (1), Christophe Yeche ${ }^{12}$, Fang-Ting Yuan ${ }^{11}$, Nadia Zakamska ${ }^{19}$ (1), Olga Zamora ${ }^{3,4}$, Gail Zasowski ${ }^{19}$, Kai Zhang ${ }^{123}{ }^{10}$, Cheng Zhao ${ }^{64}$, Gong-Bo Zhao $^{76,80}$ (1) , Zheng Zheng ${ }^{76}$ (1), Zheng Zheng ${ }^{23}$, Zhi-Min Zhou ${ }^{76}$ (D), Guangtun Zhu ${ }^{19,127}$ (D), Joel C. Zinn ${ }^{88}$ (1), and $\mathrm{Hu} \mathrm{Zou}{ }^{76}$ (iD

${ }^{1}$ Instituto de Física Teórica, (UAM/CSIC), Universidad Autónoma de Madrid, Cantoblanco, E-28049 Madrid, Spain ${ }^{2}$ Campus of International Excellence UAM+CSIC, Cantoblanco, E-28049 Madrid, Spain ${ }^{3}$ Instituto de Astrofísica de Canarias, E-38205 La Laguna, Tenerife, Spain

${ }^{4}$ Departamento de Astrofísica, Universidad de La Laguna (ULL), E-38206 La Laguna, Tenerife, Spain

${ }^{5}$ Instituto de Investigación Multidisciplinario en Ciencia y Tecnología, Universidad de La Serena, Benavente 980, La Serena, Chile

${ }^{6}$ Leibniz-Institut fuer Astrophysik Potsdam (AIP), An der Sternwarte 16, D-14482 Potsdam, Germany

${ }^{7}$ Department of Astronomy, University of Washington, Box 351580, Seattle, WA 98195, USA

${ }_{9}^{8}$ PITT PACC, Department of Physics and Astronomy, University of Pittsburgh, Pittsburgh, PA 15260, USA

${ }^{9}$ School of Physics and Astronomy, University of Nottingham, University Park, Nottingham, NG7 2RD, UK

${ }^{10}$ Unidad de Astronomía, Universidad de Antofagasta, Avenida Angamos 601, Antofagasta 1270300, Chile

${ }^{11}$ Shanghai Astronomical Observatory, Chinese Academy of Science, 80 Nandan Road, Shanghai 200030, P. R. China

${ }^{12}$ IRFU, CEA, Centre d'Etudes Saclay, F-91191 Gif-Sur-Yvette Cedex, France

${ }^{13}$ APC, University of Paris Diderot, CNRS/IN2P3, CEA/IRFU, Observatoire de Paris, Sorbonne Paris Cite, France

${ }^{14}$ Instituto de Astronomía, Universidad Nacional Autónoma de México, Apartado Postal 70-264, México D.F., 04510 Mexico

${ }^{15}$ Lawrence Berkeley National Laboratory, 1 Cyclotron Road, Berkeley, CA 94720, USA

${ }^{16}$ Universidade de São Paulo, IAG, Rua do Matão 1226, São Paulo 05508-900, Brazil

${ }^{17}$ Laboratório Interinstitucional de e-Astronomia-LIneA, Rua Gal. José Cristino 77, Rio de Janeiro, RJ-20921-400, Brazil

${ }^{18}$ Department of Physics \& Astronomy, Texas Christian University, Fort Worth, TX 76129, USA

${ }^{19}$ Center for Astrophysical Sciences, Department of Physics and Astronomy, Johns Hopkins University, 3400 North Charles Street, Baltimore, MD 21218, USA ${ }^{20}$ Department of Astronomy, Yale University, 52 Hillhouse Avenue, New Haven, CT 06511, USA

${ }^{21}$ School of Physics and Astronomy, University of St Andrews, North Haugh, St Andrews KY16 9SS, UK

${ }^{22}$ Humboldt-Universitat zu Berlin, Institut für Physik, Newtonstrasse 15, D-12589, Berlin, Germany

${ }^{23}$ Department of Physics and Astronomy, University of Utah, 115 S. 1400 E., Salt Lake City, UT 84112, USA

${ }^{24}$ Department of Physics and JINA Center for the Evolution of the Elements, University of Notre Dame, Notre Dame, IN 46556, USA

${ }^{25}$ Cavendish Laboratory, University of Cambridge, 19 J. J. Thomson Avenue, Cambridge CB3 OHE, UK

${ }^{26}$ University of Cambridge, Kavli Institute for Cosmology, Cambridge, CB3 OHE, UK
${ }^{27}$ Department of Astronomy, University of Wisconsin-Madison, 475 N. Charter St., Madison WI 53706, USA

${ }^{28}$ Department of Physics and Astronomy, Vanderbilt University, 6301 Stevenson Center, Nashville, TN, 37235, USA

${ }^{29}$ Apache Point Observatory, P.O. Box 59, Sunspot, NM 88349, USA

${ }^{30}$ Department of Astronomy, New Mexico State University, Las Cruces, NM 88003, USA

${ }^{31}$ Sternberg Astronomical Institute, Moscow State University, Moscow 119992, Russia

${ }^{32}$ Observatories of the Carnegie Institution for Science, 813 Santa Barbara St., Pasadena, CA, 91101, USA

33 Departamento de Astronomía, Universidad de Chile, Camino del Observatorio 1515, Las Condes, Santiago, Chile

${ }^{34}$ Centro de Astrofísica y Tecnologías Afines (CATA), Camino del Observatorio 1515, Las Condes, Santiago, Chile

${ }^{35}$ Center for Cosmology and Particle Physics, Department of Physics, New York University, New York, NY 10003, USA

${ }^{36}$ Aix Marseille Université, CNRS, LAM (Laboratoire d'Astrophysique de Marseille) UMR 7326, F-13388, Marseille, France

${ }^{37}$ National Optical Astronomy Observatory, 950 N Cherry Ave., Tucson, AZ 85719, USA

38 Instituto de Física y Astronomía, Universidad de Valparaíso, Av. Gran Bretaña 1111, Playa, Ancha, Casilla 5030, Chile

${ }_{39}$ Millennium Institute of Astrophysics (MAS), Monseñor Sotero Sanz 100, oficina 104, Providencia, Santiago, Chile

${ }^{40}$ Department of Astronomy and Astrophysics, University of Toronto, 50 St. George St., Toronto, ON M55 3H4, Canada

${ }^{41}$ Dunlap Institute for Astronomy \& Astrophysics, University of Toronto, 50 St. George St., Toronto, ON M5S 3H4, Canada

${ }^{42}$ Department of Astronomy and Astrophysics, Eberly College of Science, The Pennsylvania State University, 525 Davey Laboratory, University Park, PA 16802, USA

${ }^{43}$ Institute for Gravitation and the Cosmos, Pennsylvania State University, University Park, PA 16802, USA

${ }^{44}$ Department of Physics, The Pennsylvania State University, University Park, PA 16802, USA

${ }^{45}$ Kavli IPMU (WPI), UTIAS, The University of Tokyo, Kashiwa, Chiba 277-8583, Japan

${ }^{46}$ Departamento de Física Matemática, Instituto de Física, Universidade de São Paulo, CP 66318, CEP 05314-970, São Paulo, SP, Brazil

${ }^{47}$ Sub-department of Astrophysics, Department of Physics, University of Oxford, Denys Wilkinson Building, Keble Road, Oxford OX1 3RH, UK

${ }_{49}$ New York University Abu Dhabi, P.O. Box 129188, Abu Dhabi, United Arab Emirates

${ }^{49}$ Korea Institute for Advanced Study, 85 Hoegiro, Dongdaemun-gu, Seoul 02455, Korea

${ }^{50}$ Departamento de Física, Centro de Ciências Naturais e Exatas, Universidade Federal de Santa Maria, 97105-900, Santa Maria, RS, Brazil

${ }^{51}$ Max-Planck-Institut für extraterrestrische Physik, Postfach 1312, Giessenbachstraße, D-85741 Garching, Germany

${ }_{52}$ Departamento de Astronomía, Universidad de Concepción, Casilla 160-C, Concepción, Chile

${ }^{53}$ Center for Astrophysics and Space Astronomy, Department of Astrophysical and Planetary Sciences, University of Colorado, 389 UCB, Boulder, CO 80309, USA

${ }^{54}$ Instituto de Física, Universidade Federal do Rio Grande do Sul, Campus do Vale, Porto Alegre, RS, 91501-907, Brazil

55 Aix Marseille Université, CNRS/IN2P3, Centre de Physique des Particules de Marseille, UMR 7346, 13288, Marseille, France

${ }^{56}$ Department of Physics and Astronomy, Western Washington University, 516 High Street, Bellingham, WA 98225, USA

${ }^{57}$ Department of Physics and McWilliams Center for Cosmology, Carnegie Mellon University, 5000 Forbes Avenue, Pittsburgh, PA 15213, USA

58 Observatório Nacional, 77 Rua General José Cristino, Rio de Janeiro, 20921-400, Brazil

${ }^{59}$ Department of Astronomy, University of Virginia, Charlottesville, VA 22904-4325, USA

${ }^{60}$ Laboratoire d'Astrophysique, École Polytechnique Fédérale de Lausanne, 1015 Lausanne, Switzerland

${ }^{61}$ Department of Physics, Geology, and Engineering Tech, Northern Kentucky University, Highland Heights, KY 41099, USA

${ }^{62}$ Centro de Investigaciones de Astronomía, AP 264, Mérida 5101-A, Venezuela 
${ }^{63}$ McDonald Observatory, The University of Texas at Austin, 1 University Station, Austin, TX 78712, USA

${ }^{64}$ Tsinghua Center for Astrophysics and Department of Physics, Tsinghua University, Beijing 100084, P. R. China

${ }^{65}$ Harvard-Smithsonian Center for Astrophysics, 60 Garden St., MS 20, Cambridge, MA 02138, USA

${ }^{66}$ School of Physics and Astronomy, University of Birmingham, Edgbaston, Birmingham B15 2TT, UK

${ }^{67}$ Stellar Astrophysics Centre, Department of Physics and Astronomy, Aarhus University, Ny Munkegade 120, DK-8000 Aarhus C, Denmark ${ }^{68}$ European Southern Observatory, Karl-Schwarzschild-Str. 2, D-85748 Garching, Germany

${ }^{69}$ Université Lyon 1, Observatoire de Lyon, Centre de Recherche Astrophysique de Lyon and École Normale Supérieure de Lyon, 9 avenue Charles André, F-69230 Saint-Genis Laval, France

${ }^{70}$ Steward Observatory, University of Arizona, 933 North Cherry Ave., Tucson, AZ 85721, USA

${ }^{71}$ Institut UTINAM, CNRS-UMR6213, OSU THETA, Université Bourgogne-Franche-Comté, 41bis avenue de l'Observatoire, 25010 Besançon Cedex, France

${ }^{72}$ Space Telescope Science Institute, 3700 San Martin Dr., Baltimore, MD 21218, USA

${ }^{73}$ CSRA Inc., 3700 San Martin Dr., Baltimore, MD 21218, USA

${ }^{74}$ Department of Physics \& Astronomy, University of Iowa, Iowa City, IA 52245, USA

${ }^{75}$ Laboratoire AIM, CEA/DRF-CNRS-Univ. Paris Diderot-IRFU/SAp, Centre de Saclay, F-91191 Gif-sur-Yvette Cedex, France

${ }^{76}$ National Astronomy Observatories, Chinese Academy of Science, 20A Datun Rd., Chaoyang District, Beijing, 100012, P. R. China

${ }^{77}$ Sorbonne Universités, UPMC Univ Paris 06, UMR 7095, Institut d'Astrophysique de Paris, 98 bis boulevard Arago, F-75014, Paris, France

${ }^{78}$ Laboratoire de Physique Nucléaire et de Hautes Energies, Université Pierre et Marie Curie, 4 Place Jussieu, F-75005 Paris, France

${ }^{79}$ Osservatorio Astronomico di Padova-INAF, Vicolo dell'Osservatorio 5, I-35122, Padova, Italy

${ }^{80}$ Institute of Cosmology \& Gravitation, University of Portsmouth, Dennis Sciama Building, Portsmouth, PO1 3FX, UK

${ }^{81}$ DePauw University, Greencastle, IN 46135, USA

${ }^{82}$ LPNHE, CNRS/IN2P3, Université Pierre et Marie Curie Paris 6, Université Denis Diderot Paris 7, 4 place Jussieu, 75252 Paris CEDEX, France

${ }^{83}$ Department of Astronomy, Case Western Reserve University, Cleveland, OH 44106, USA

${ }_{85}^{84}$ Max Planck Institute for Solar System Research, Justus-von-Liebig-Weg 3, 37077 Goettingen, Germany

${ }^{85}$ Sydney Institute for Astronomy (SIfA), School of Physics, University of Sydney, NSW 2006, Australia

${ }^{86}$ SETI Institute, 189 Bernardo Ave., Mountain View, CA 94043, USA

${ }^{87}$ Rice University, 6100 Main St., Houston, TX 77005, USA

${ }^{88}$ Department of Astronomy, The Ohio State University, 140 W. 18th Ave., Columbus, OH 43210, USA

${ }^{89}$ Center for Cosmology and AstroParticle Physics, The Ohio State University, 191 W. Woodruff Ave., Columbus, OH 43210, USA

${ }_{90}$ Max-Planck-Institut fuer Astrophysik, Karl-Schwarzschild-Str. 1, D-85748 Garching, Germany

${ }_{91}$ Institute for Astronomy, University of Vienna, Türkenschanzstrasse 17, A-1180 Vienna, Austria

92 Department of Physics and Astronomy, University of California, Irvine, Irvine, CA 92697, USA

${ }^{93}$ Instituto de Astrofísica, Pontificia Universidad Católica de Chile, Av. Vicuna Mackenna 4860, 782-0436 Macul, Santiago, Chile

${ }^{94}$ Astrophysics Research Institute, Liverpool John Moores University, IC2, Liverpool Science Park, 146 Brownlow Hill, Liverpool L3 5RF, UK ${ }^{95}$ CSIC, Serrano, 117, E-28006, Madrid, Spain

${ }^{96}$ Space Science Institute, 4750 Walnut St., Suite 205, Boulder, CO 80301, USA

${ }^{97}$ ELTE Gothard Astrophysical Observatory, H-9704 Szombathely, Szent Imre herceg st. 112, Hungary

${ }^{98}$ Departamento de Ciencias Fisicas, Universidad Andres Bello, Sazie 2212, Santiago, Chile

${ }^{99}$ Max-Planck-Institut für Astronomie, Konigstuhl 17, D-69117 Heidelberg, Germany

${ }^{100}$ LESIA, UMR 8109 , Université Pierre et Marie Curie, Université Denis Diderot, Observatoire de Paris, F-92195 Meudon Cedex, France

${ }_{101} 1$ Department of Physics and Astronomy, University of Wyoming, Laramie, WY 82071, USA

${ }_{102}$ Department of Physics and Astronomy, University of Alabama, Tuscaloosa, AL 35487-0324, USA

${ }^{103}$ Institute for Astronomy, University of Edinburgh, Royal Observatory, Edinburgh EH9 3HJ, UK

${ }^{104}$ Université Paris 6 et CNRS, Institut d'Astrophysique de Paris, 98bis blvd. Arago, F-75014 Paris, France

${ }^{05}$ Instituto de Astrofísica de Andalucía (CSIC), Glorieta de la Astronomía, E-18080 Granada, Spain

${ }^{106}$ Department of Astronomy and Astrophysics, University of California, Santa Cruz and UC Observatories, Santa Cruz, CA, 95064, USA

${ }^{107}$ Dipartimento di Fisica e Astronomia, Università di Padova, Vicolo dell'Osservatorio 2, I-35122 Padova, Italy

${ }^{108}$ Departamento de Fisica y Astronomia, Universidad de La Serena, Cisternas 1200, La Serena, 0000-0002-1379-4204, Chile

${ }^{109}$ Instituto de Astronomía, Universidad Nacional Autónoma de México, Unidad Académica en Ensenada, Ensenada BC 22860, Mexico

${ }^{110}$ Department of Astronomy and Space Science, Sejong University, Seoul 143-747, Korea

${ }^{111}$ Observatoire de la Côte d'Azur, Laboratoire Lagrange, F-06304 Nice Cedex 4, France

112 Department of Physics and Astronomy, Ohio University, Clippinger Labs, Athens, OH 45701, USA

${ }_{113}$ Institute of Space Sciences (IEEC-CSIC), Carrer de Can Magrans, E-08193, Barcelona, Spain

${ }^{114}$ University of Texas at Austin, McDonald Observatory, McDonald Observatory, TX, 79734, USA

115 Brookhaven National Laboratory, Upton, NY 11973, USA

${ }^{116}$ National Optical Astronomy Observatories, Tucson, AZ, 85719, USA

117 School of Physics, The University of New South Wales, Sydney NSW 2052, Australia

${ }^{118}$ Department of Astrophysical Sciences, Princeton University, Princeton, NJ 08544, USA

${ }^{119}$ Instituto de Física, Universidad Nacional Autónoma de México, Apdo. Postal 20-364, Mexico

${ }^{120}$ Department of Physical Sciences, The Open University, Milton Keynes MK7 6AA, UK

${ }^{121}$ Department of Physics, University of North Carolina Asheville, One University Heights, Asheville, NC 28804, USA

122 Physics Department, Austin College, Sherman, TX 75092, USA

${ }^{123}$ Department of Physics and Astronomy, University of Kentucky, 505 Rose St., Lexington, KY 40506, USA

${ }_{124}$ Department of Physics, Bridgewater College, 402 E. College St., Bridgewater, VA 22812, USA

125 Academia Sinica Institute of Astronomy and Astrophysics, P.O. Box 23-141, Taipei 10617, Taiwan

Received 2016 August 8; revised 2017 August 7; accepted 2017 August 27; published 2017 December 8

\begin{abstract}
The fourth generation of the Sloan Digital Sky Survey (SDSS-IV) began observations in 2014 July. It pursues three core programs: the Apache Point Observatory Galactic Evolution Experiment 2 (APOGEE-2), Mapping Nearby Galaxies at APO (MaNGA), and the Extended Baryon Oscillation Spectroscopic Survey (eBOSS). As well as its core program, eBOSS contains two major subprograms: the Time Domain Spectroscopic Survey (TDSS) and the
\end{abstract}

\footnotetext{
126 la Caixa-Severo Ochoa Scholar.

127 Hubble Fellow.
} 
SPectroscopic IDentification of ERosita Sources (SPIDERS). This paper describes the first data release from SDSS-IV, Data Release 13 (DR13). DR13 makes publicly available the first 1390 spatially resolved integral field unit observations of nearby galaxies from MaNGA. It includes new observations from eBOSS, completing the Sloan Extended QUasar, Emission-line galaxy, Luminous red galaxy Survey (SEQUELS), which also targeted variability-selected objects and X-ray-selected objects. DR13 includes new reductions of the SDSS-III BOSS data, improving the spectrophotometric calibration and redshift classification, and new reductions of the SDSS-III APOGEE-1 data, improving stellar parameters for dwarf stars and cooler stars. DR13 provides more robust and precise photometric calibrations. Value-added target catalogs relevant for eBOSS, TDSS, and SPIDERS and an updated red-clump catalog for APOGEE are also available. This paper describes the location and format of the data and provides references to important technical papers. The SDSS web site, http://www.sdss.org, provides links to the data, tutorials, examples of data access, and extensive documentation of the reduction and analysis procedures. DR13 is the first of a scheduled set that will contain new data and analyses from the planned $\sim 6 \mathrm{yr}$ operations of SDSS-IV.

Key words: atlases - catalogs - surveys

\section{Introduction}

The Sloan Digital Sky Survey (SDSS) has been observing the universe using the $2.5 \mathrm{~m}$ Sloan Foundation Telescope (Gunn et al. 2006) at Apache Point Observatory (APO) for over 15 yr. The goal of the original survey (2000-2005; York et al. 2000) was to map large-scale structure with five-band imaging over $\sim \pi$ sr of the sky and spectra of $\sim 10^{6}$ galaxies and $\sim 10^{5}$ quasars. This program was accomplished using a driftscan camera (Gunn et al. 1998) and two fiber-fed optical $R \sim 1800$ spectrographs (Smee et al. 2013), each with 320 fibers.

The imaging and spectroscopy goals were not entirely fulfilled in the initial $5 \mathrm{yr}$ period, and thus SDSS-I was followed by SDSS-II (2005-2008; Abazajian et al. 2009). Its first goal was to complete the planned initial large-scale structure redshift survey as the Legacy program. It added SEGUE (Sloan Extension for Galactic Understanding and Exploration; Yanny et al. 2009), a spectroscopic survey focused on stars, and imaged an average of once every 5 days a $\sim 200 \mathrm{deg}^{2}$ area along the celestial equator with repeated scans in SDSS-I ("Stripe 82"), to search for Type Ia supernovae and other transients (Frieman et al. 2008).

The success of SDSS as a cosmological probe, particularly the detection of the clustering of luminous red galaxies (LRGs) on the $100 \mathrm{~h}^{-1} \mathrm{Mpc}$ scale expected from baryon acoustic oscillations (BAOs; Eisenstein et al. 2005), led to the conception and implementation of BOSS (Baryon Oscillation Spectroscopic Survey; Dawson et al. 2013) as the flagship program in the third version of the survey, SDSS-III (2008-2014; Eisenstein et al. 2011). As part of BOSS, SDSS-III imaged additional areas in the part of the south Galactic cap visible from the Northern Hemisphere. At the conclusion of these observations, the SDSS imaging camera was retired and is now part of the permanent collection of the Smithsonian National Air and Space Museum. ${ }^{128}$ During the summer shutdown in 2009, the original SDSS spectrographs were replaced by new, more efficient spectrographs to be used by BOSS. The BOSS spectrographs featured expanded wavelength coverage $(3560 \AA<\lambda<10400 \AA)$, new CCD detectors with improved read noise, smaller pixels $(15 \mu \mathrm{m})$, and improved quantum efficiency, as well as VPH gratings instead of the original replicated surface relief gratings (Smee et al. 2013). The two spectrographs were now fed by 500 fibers

\footnotetext{
$\overline{128}$ https://airandspace.si.edu/collection-objects/camera-imaging-digitalsloan-digital-sky-survey-ccd-array
}

each so that the desired number of redshifts could be reached in the planned survey lifetime.

During the first year of SDSS-III (2008-2009), the SEGUE2 survey (C. Rockosi et al. 2017, in preparation) used the original SDSS spectrographs to observe additional Milky Way halo fields to target distant halo samplers and trace substructure. In SDSS-III all bright time could be used for scientific observations with the arrival of two new instruments. MARVELS (Multi-object APO Radial Velocity Exoplanet Large-area Survey; Paegert et al. 2015; Thomas et al. 2016) used a novel multiplexing interferometer to observe 60 stars simultaneously to search for radial velocity variations caused by hot Jupiters and close brown dwarf companions. APOGEE (Apache Point Observatory Galactic Evolution Experiment; Majewski et al. 2017) used a 300-fiber, $R \sim 22,000 H$-band spectrograph (Majewski et al. 2017; J. Wilson et al. 2017, in preparation) to measure stellar parameters, chemical abundances, and radial velocities, mainly for red giants (Zasowski et al. 2013).

Since routine operations started in 2000, there have been 13 public data releases. All data releases are cumulative, rereleasing the best reduction of all previously taken data. The most recent of these was Data Release 12 (Alam et al. 2015), which contained all of the SDSS-III data, as well as the rereduced data from SDSS-I and SDSS-II. SDSS-I to SDSS-III imaged 14,555 $\mathrm{deg}^{2}$ in the five filters (Fukugita et al. 1996; Doi et al. 2010). Most of the sky was surveyed once or twice, but regions in Stripe 82 were observed between 70 and 90 times. By the time of their retirement, the SDSS spectrographs had obtained $R \sim 1800$ optical spectra for 860,836 galaxies, 116,003 quasars, and 521,990 stars. With the BOSS spectrographs, the survey has added data with similar resolution for 1,372,737 galaxies, 294,512 quasars, and 247,216 stars. APOGEE has contributed high-resolution IR spectra of 156,593 stars. MARVELS had observed 3233 stars with at least 16 epochs of radial velocity measurements.

The success of the previous SDSS releases and the continuing importance of the wide-field, multiplexing capability of the Sloan Foundation Telescope motivated the organization of the fourth phase of the survey, SDSS-IV (Blanton et al. 2017). SDSS-IV extends SDSS observations to many fibers covering the spatial extent of nearby galaxies, to new redshift regimes, and to the parts of the Milky Way and dwarf galaxies that are only visible from the Southern Hemisphere. The MaNGA (Mapping Nearby Galaxies at APO) survey studies galaxy formation and evolution across a 
wide range of masses and morphological types by observing a substantial portion of the optical spatial extent of $\sim 10^{4}$ galaxies (Bundy et al. 2015). It accomplishes this goal by employing 17 bundles ranging in size between 19 and 127 fibers to cover targets selected from an extended version of the NASA-Sloan Atlas ${ }^{129}$ and 12 bundles of seven fibers for calibration stars. These integral field units (IFUs) feed the BOSS spectrographs, providing information on the properties of gas and stars in galaxies out to $1.5-2.5$ effective radii $\left(R_{e}\right)$.

Another survey, eBOSS (extended Baryon Oscillation Spectroscopic Survey; Dawson et al. 2016), shares the dark time equally with MaNGA. eBOSS will measure with percentlevel precision the distance-redshift relation with $\mathrm{BAO}$ in the clustering of matter over the relatively unconstrained redshift range $0.6<z<2.2$. This redshift range probes the universe during its transition from matter dominated to dark energy dominated. Multiple measurements of the angular diameter distance $\left(d_{A}(z)\right)$ and Hubble parameter $(H(z))$ from BAO over the redshifts covered by eBOSS are therefore crucial for understanding the nature of dark energy. eBOSS will use spectroscopic redshifts from more than 400,000 LRGs and nearly 200,000 emission-line galaxies (ELGs) to extend the final BOSS galaxy clustering measurements (Alam et al. 2017) by providing two new BAO distance measurements over the redshift interval $0.6<z<1.1$. Roughly 500,000 spectroscopically confirmed quasars will be used as tracers of the underlying matter density field at $0.9<z<2.2$, providing the first measurements of BAO in this redshift interval. Finally, the Ly $\alpha$ forest imprinted on approximately 120,000 new quasar spectra will give eBOSS an improved BAO measurement over that achieved by BOSS (Delubac et al. 2015; Bautista et al. 2017). The three new tracers will provide BAO distance measurements with a precision of $1 \%$ at $z=0.7$ (LRG), $2 \%$ at $z=0.85$ (ELG), and $2 \%$ at $z=1.5$ (quasar), while the enhanced Ly $\alpha$ forest sample will improve BOSS constraints by a factor of 1.4. Furthermore, the clustering from eBOSS tracers will allow new measurements of redshift-space distortions (RSDs), non-Gaussianity in the primordial density field, and the summed mass of neutrino species. Extensively observing these redshift ranges for the first time in SDSS required re-evaluation of targeting strategies. Preliminary targeting schemes for many of these classes of objects were tested as part of SEQUELS (Sloan Extended QUasar, Emission-line galaxy, Luminous red galaxy Survey), which used 126 plates observed across SDSSIII and SDSS-IV. DR13 includes all SEQUELS data, giving the largest SDSS sample to date of spectra targeting intermediate redshift ranges. SDSS-IV also allocated a significant number of fibers on the eBOSS plates to two additional dark-time programs. TDSS (Time Domain Spectroscopic Survey; Morganson et al. 2015) seeks to understand the nature of celestial variables by deliberately targeting objects that vary in combined SDSS DR9 and Pan-STARRS1 data (PS1; Kaiser et al. 2002).

A large number of the likely quasar targets so selected are also targeted by the main eBOSS algorithms and therefore meet the goals of both surveys. TDSS-only targets fill $\sim 10$ spectra per square degree. The main goal of the SPIDERS (Spectroscopic Identification of eROSITA Sources) survey is to characterize a subset of X-ray sources identified by eROSITA (extended Roentgen Survey with an Imaging Telescope Array;

${ }^{129}$ http://www.nsatlas.org
Predehl et al. 2014). Until the first catalog of eROSITA sources is available, SPIDERS will target sources from the RASS (ROSAT All Sky Survey; Voges et al. 1999) and XMM (X-ray Multi-mirror mission; Jansen et al. 2001). SPIDERS will also obtain on average $\sim 10$ spectra per square degree over the course of SDSS-IV, but the number of fibers per square degree on a plate is weighted toward the later years to take advantage of the new data from eROSITA.

In bright time at APO, APOGEE-2, the successor to APOGEE (hereafter APOGEE-1) in SDSS-IV, will continue its survey of the Milky Way stellar populations. Critical areas of the Galaxy, however, cannot be observed from APO, including the more distant half of the Galactic bar, the fourth quadrant of the disk, and important dwarf satellites of the Milky Way, such as the Magellanic Clouds and some dwarf spheroidals. SDSS-IV will for the first time include operations outside of APO as the result of Carnegie Observatories and the Chilean Participation Group joining the collaboration. A second APOGEE spectrograph is being constructed for installation on the $2.5 \mathrm{~m}$ du Pont Telescope (Bowen \& Vaughan 1973) at Las Campanas Observatory (LCO) near La Serena, Chile. When APOGEE-2S begins survey operations in 2017, approximately 300 survey nights on the du Pont Telescope will be used to extend the APOGEE-2 survey to the Southern Hemisphere.

Data Release 13 (DR13) is the first data release for SDSSIV, which will have regular public, documented data releases, in keeping with the philosophy of SDSS since its inception. In this paper, we describe the data available in DR13, focusing on the data appearing here for the first time. We present overall descriptions of the sample sizes and targeting and provide a detailed bibliography of the technical papers available to understand the data and the surveys in more detail. These technical papers and the SDSS web site (http://www.sdss.org) contain critical information about these data, which here is only summarized.

\section{Overview of the Survey Landscape}

The release of DR13 coincides with the arrival of an astonishingly rich set of data from ongoing and recently completed surveys outside of SDSS. Blanton et al. (2017), Bundy et al. (2015), and Majewski et al. (2017) describe how the SDSS-IV surveys compare in survey strategy, size, and data within the wider arena of spectroscopic surveys. We cite here some key science results for these works to complement our brief history of SDSS.

Spectroscopic redshift surveys of large-scale structure have resulted in BAO measurements over a range of redshifts. The BAO signal has been detected at lower redshift $(z \sim 0.1)$ from measurements of $\sim 75,000$ galaxies in the $6 \mathrm{dF}$ Galaxy Redshift Survey (Beutler et al. 2011). WiggleZ measured the BAO signal at similar redshifts to BOSS based on redshifts of $\sim 225,000$ galaxies. The final WiggleZ results for the $1 \mathrm{D}$ BAO peak (Kazin et al. 2014) and the 2D BAO peak (Hinton et al. 2017) at $z=0.44,0.6$, and 0.73 agree with the BOSS results. Still under way is the VIMOS Public Extragalactic Redshift Survey, which focuses on higher redshifts than previous work $(0.5<z<1.2)$ and will overlap in part with eBOSS (Guzzo et al. 2014).

When the MaNGA survey began, two pioneering and highly influential IFU surveys of hundreds of galaxies were being completed: CALIFA (Sánchez et al. 2012) and ATLAS ${ }^{3 \mathrm{D}}$ 
(Cappellari et al. 2011). ATLAS ${ }^{3 \mathrm{D}}$ observed 260 morphologically classified early-type galaxies (ETGs) within $40 \mathrm{Mpc}$ at optical, radio, and infrared wavelengths, including optical IFU observations with the PPAK integral field unit instrument. CALIFA released IFU data from SAURON on 667 galaxies in its third and final data release (Sánchez et al. 2016). These galaxies spanned a range of morphologies from irregular to elliptical, over $7 \mathrm{mag}$ in luminosity, and over $3 \mathrm{mag}$ in $u-z$ colors. The increase in the number of galaxies with IFU data led to many pivotal discoveries regarding galaxy evolution, including advances in our knowledge of the origin and heating sources of gas in ETGs, gas and stellar abundance gradients in galaxies, and the star formation rate and age of stellar populations, highlighted below.

Davis et al. (2011) used ATLAS ${ }^{3 \mathrm{D}}$ to investigate the origins of the gas in slow- and fast-rotating ETGs in different environments. Overall, ETGs are poor in atomic and molecular gas and therefore lack much star formation, and the cause of this transformation is critical to understanding galaxy formation. Alatalo et al. (2011) identified one mechanism for gas depletion in the ATLAS ${ }^{3 \mathrm{D}}$ galaxy NGC 1266, in the form of a strong molecular wind from the nucleus, likely powered by a hidden AGN. Kehrig et al. (2012) suggested an AGN as an energy source for the elongated ionized gas observed by CALIFA in NGC 5966. The CALIFA data also showed that NGC 5966 and its fellow ETG, NGC 6762, had extended line emission best explained by heating from post-asymptotic giant branch (post-AGB) stars. Gomes et al. (2016) studied 32 ETGs in CALIFA and found extended $\mathrm{H} \alpha$ emission that fell into two broad classes based on whether the intensity of emission is flat or increasing with radius.

The ages of stellar populations across CALIFA's wide range of Hubble types show that galaxies with stellar masses $\gtrsim 5 \times 10^{9} M_{\odot}$ form from the inside out, with the more massive galaxies having older stellar populations in each scaled radial bin (Pérez et al. 2013). Cano-Díaz et al. (2016) showed that the relationship between star formation rate (SFR) and stellar mass that held for entire star-forming galaxies was also true for spatially resolved regions within a galaxy. In another intriguing clue to the relation between small and large scales, Sánchez et al. (2014) found that the H II regions in 306 CALIFA galaxies have a characteristic disk oxygen abundance gradient when scaled to its effective radius.

Another large IFU survey, the SAMI survey (Bryant et al. 2015), is currently under way at the Anglo-Australian Telescope and will ultimately observe 3400 galaxies. The Early Data Release (Allen et al. 2015) provided data for 107 galaxies, while the first major data release (Green et al. 2017) includes 772 galaxies. Science results from the SAMI survey so far include characterizing the galactic winds or extended diffuse ionizing gas in edge-on disk galaxies (Ho et al. 2016), mapping the quenching of star formation proceeding from the outside in in dense environments (Schaefer et al. 2017), and identifying stellar mass as the main variable affecting the fraction of ETGs that are slow rotators (Brough et al. 2017).

While APOGEE-2 is currently the only infrared stellar spectroscopic survey, there are several Galactic stellar surveys of similar scope observing at optical wavelengths. The RAVE survey (Steinmetz et al. 2006) completed observations in 2013 with $R \sim 7500$ spectra of $\sim 450,000$ bright stars in the Ca II triplet region released in Data Release 5 (Kunder et al. 2017). RAVE's primary goal was to obtain spectroscopic measurements for stars with exquisite Gaia proper-motion and parallax measurements. The $\sim 250,000$ stars in DR5 that have proper motions and parallaxes in the first Gaia data release (Gaia Collaboration et al. 2016) now make this work possible in both the disk (e.g., Robin et al. 2017) and halo (e.g., Helmi et al. 2017). Among the many results published prior to the Gaia results were measurements of the local escape velocity (e.g., Smith et al. 2007; Piffl et al. 2014), detection of a "wobbly" galaxy from asymmetric velocities both radially and across the disk (Siebert et al. 2011; Williams et al. 2013), and the observation of extra-tidal stars from Galactic globular clusters (e.g., Kunder et al. 2014; Fernández-Trincado et al. 2015; Anguiano et al. 2016).

The Gaia-ESO survey recently completed observations of $\sim 10^{5}$ cluster and field stars at either $R \sim 20,000$ or $R \sim 47,000$ (Gilmore et al. 2012). The Gaia-ESO collaboration has presented important results about the nature of several Galactic components, including the abundances and kinematics in the bulge (e.g., Howes et al. 2014; Recio-Blanco et al. 2017; Rojas-Arriagada et al. 2017), characterizing the accreted component of the halo and (if any) disk (e.g., Ruchti et al. 2015), and measuring radial abundance gradients in the open cluster population (e.g., Jacobson et al. 2016; Magrini et al. 2017).

The LAMOST Galactic survey is obtaining spectra, 4000 at a time, at a similar resolution to SEGUE (Zhao et al. 2012). Data $^{130}$ Release 3 includes 5.75 million spectra and stellar parameters for $>3$ million stars. LAMOST has an ideal view of the field observed for four years by the Kepler satellite, and several groups have used the $\sim 50,000$ stars observed in the Kepler field to characterize its stellar populations (e.g., Dong et al. 2014; Frasca et al. 2016; Ren et al. 2016; Chang et al. 2017; Dong et al. 2017). Other work based on LAMOST spectra includes measurements of stellar activity (e.g., Fang et al. 2016) and identification of important subclasses of objects from ultra-metal-poor stars (e.g., Li et al. 2015) and metalliclined Am stars (e.g., Hou et al. 2015).

The GALAH survey (De Silva et al. 2015), which started operations in 2014, released spectra for over 200,000 stars observed with the HERMES spectrograph $(R \sim 28,000)$ at the Anglo-Australian Telescope (Martell et al. 2017). When the survey is completed, spectra of $\sim 10^{6}$ stars in four optical windows accessing 29 elements are expected.

\section{Scope of Data Release 13}

SDSS-IV has been operating since 2014 July. DR13 contains data gathered between 2014 July and 2015 July and is summarized in Table 1. The categories under MaNGA galaxies are described in Section 5. The SEQUELS targeting flags are listed and described in Alam et al. (2015). Figures 1-3 show the sky coverage of the MaNGA, eBOSS, and APOGEE-2 surveys, respectively. In the subsequent sections, we discuss each survey's data in detail, but briefly DR13 includes the following:

1. Reduced data for the 82 MaNGA galaxy survey plates, yielding 1390 reconstructed 3D data cubes for 1351 unique galaxies, that were completed by 2015 July. Rowstacked spectra (RSS) and raw data are also included.

${ }^{130}$ dr3.lamost.org 
Table 1

Reduced Spectroscopic Data in DR13

\begin{tabular}{|c|c|c|}
\hline Target Category & \# DR12 & \# DR12+13 \\
\hline \multicolumn{3}{|l|}{ MaNGA main galaxy sample: } \\
\hline PRIMARY_v1_2 & 0 & 600 \\
\hline SECONDARY_v1_2 & 0 & 473 \\
\hline COLOR-ENHANCED_v1_2 & 0 & 216 \\
\hline MaNGA ancillary galaxies $^{\mathrm{a}}$ & 0 & 31 \\
\hline MaNGA Other & 0 & 62 \\
\hline \multicolumn{3}{|l|}{ SEQUELS } \\
\hline LRG_IZW & 11781 & 21271 \\
\hline LRG_RIW & 11691 & 20967 \\
\hline QSO_EBOSS_CORE & 19455 & 33367 \\
\hline QSO_PTF & 13227 & 22609 \\
\hline QSO_REOBS & 1357 & 2238 \\
\hline QSO_EBOSS_KDE & 11836 & 20474 \\
\hline QSO_EBOSS_FIRST & 293 & 519 \\
\hline QSO_BAD_BOSS & 59 & 95 \\
\hline QSO_BOSS_TARGET & 59 & 95 \\
\hline DR9_CALIB_TARGET & 28594 & 49765 \\
\hline SPIDERS_RASS_AGN & 162 & 275 \\
\hline SPIDERS_RASS_CLUS & 1533 & 2844 \\
\hline TDSS_A & 9412 & 17394 \\
\hline TDSS_FES_DE & 40 & 70 \\
\hline TDSS_FES_DWARFC & 19 & 29 \\
\hline TDSS_FES_NQHISN & 73 & 148 \\
\hline TDSS_FES_MGII & 1 & 2 \\
\hline TDSS_FES_VARBAL & 55 & 103 \\
\hline SEQUELS_PTF_VARIABLE & 700 & 1153 \\
\hline \multicolumn{3}{|l|}{ APOGEE-2 } \\
\hline All Stars & 164562 & 164562 \\
\hline NMSU $1 \mathrm{~m}$ stars & 894 & 894 \\
\hline Telluric stars & 17293 & 17293 \\
\hline Commissioning stars & 11917 & 11917 \\
\hline
\end{tabular}

Note.

${ }^{a}$ Many MaNGA ancillary targets were also targeted as part of the main galaxy sample and are counted twice in this table.

2. Reduced BOSS spectrograph data for an additional 60 SEQUELS plates, completing the SEQUELS program. The total number of SEQUELS plates released in DR12 and DR13 is 126. These plates provide a complete footprint covering roughly $400 \mathrm{deg}^{2}$ that will not be revisited in eBOSS. The targets include a superset of the eBOSS LRG and quasar samples, a sample of variabilityselected point sources at a much higher density than in TDSS, and new X-ray-selected objects selected by similar criteria to targets in SPIDERS.

3. The reduced data for five BOSS plates at low declination in the SGC. These plates were drilled during SDSS-III but not observed owing to insufficient open-dome time when they were observable. The plates were observed early in SDSS-IV to fill in the footprint in this region.

4. Spectroscopic data from BOSS processed with a new version of the data reduction pipeline, which results in less-biased flux values.

5. All APOGEE-1 data re-reduced with improved telluric subtraction and analyzed with an improved pipeline and synthetic grid, including adding rotational broadening as a parameter for dwarf spectra.

6. New species (C I, P, Ti II, Co, Cu, Ge, and Rb) with reported abundances for the APOGEE-1 sample.
7. Stellar parameters for APOGEE-1 stars with cooler effective temperatures $\left(T_{\text {eff }}<3500 \mathrm{~K}\right)$, derived by an extension of the grid of synthetic spectra using MARCS (Gustafsson et al. 2008) model atmospheres.

8. Recalibrated SDSS imaging catalogs, using the hypercalibration to PanSTARRS 1 implemented by Finkbeiner et al. (2016).

9. Valued-added catalogs, see Table 2. More detail and direct links to the catalogs and their data models can be found at http://www.sdss.org/dr13/data_access/vac.

10. The most recent reductions of all data from previous iterations of SDSS are included as a matter of course. For MARVELS data, these data are the same as in DR12; for SEGUE and SEGUE-2, the same as in DR9.

DR13 contains only a subset of the reduced or raw data for all surveys taken between 2014 July and 2015 July. The first 2 yr of eBOSS data are needed before useful cosmological constraints can be extracted. APOGEE-2 is using the first year of SDSS-IV data to work on science verification and targeting optimization for new classes of targets and new survey strategies. Both of these surveys will release more extensive new data in Data Release 14.

\section{Data Distribution}

The data for DR13 are distributed through the same mechanisms available in DR12, with some significant changes to the environment used to access the catalog data (see below). Raw and processed image and spectroscopic data are available, as before, through the Science Archive Server (SAS, data.sdss. org/sas/dr13), and also for imaging data, optical spectra, and APOGEE IR spectra through an interactive web application (dr13.sdss.org, available soon). The catalogs of photometric, spectroscopic, and derived quantities are available through the Catalog Archive Server or CAS (Thakar et al. 2008; Thakar 2008) via two primary modes of access: browser-based queries of the database are available through the SkyServer Web application (http://skyserver.sdss.org) in synchronous mode, and more advanced and extensive querying capabilities are available through CasJobs (http://skyserver.sdss.org/ casjobs) in asynchronous or batch mode that allows timeconsuming queries to be run in the background ( $\mathrm{Li} \&$ Thakar 2008).

The CAS is now part of the new SciServer (http://www. sciserver.org/) collaborative science framework that allows users single-sign-on access to a suite of collaborative datadriven science services that include the classic SDSS services of SkyServer and CasJobs. These services are essentially unchanged in their user interfaces but have acquired powerful new capabilities and undergone fundamental re-engineering to make them interoperable and applicable to other science domains. New services are also available to users once they register on the SciServer portal, and these services work seamlessly with the existing tools. Most notable among the new offerings are SciDrive, a distributed DropBox-like file store; SkyQuery, a federated cross-matching service that compares and combines data from a collection (federation) of multiwavelength archives (SkyNodes); and SciServer Compute, a powerful new system for uploading complex analysis scripts as Jupyter notebooks (using Python, MatLab or R) running in Docker containers. 


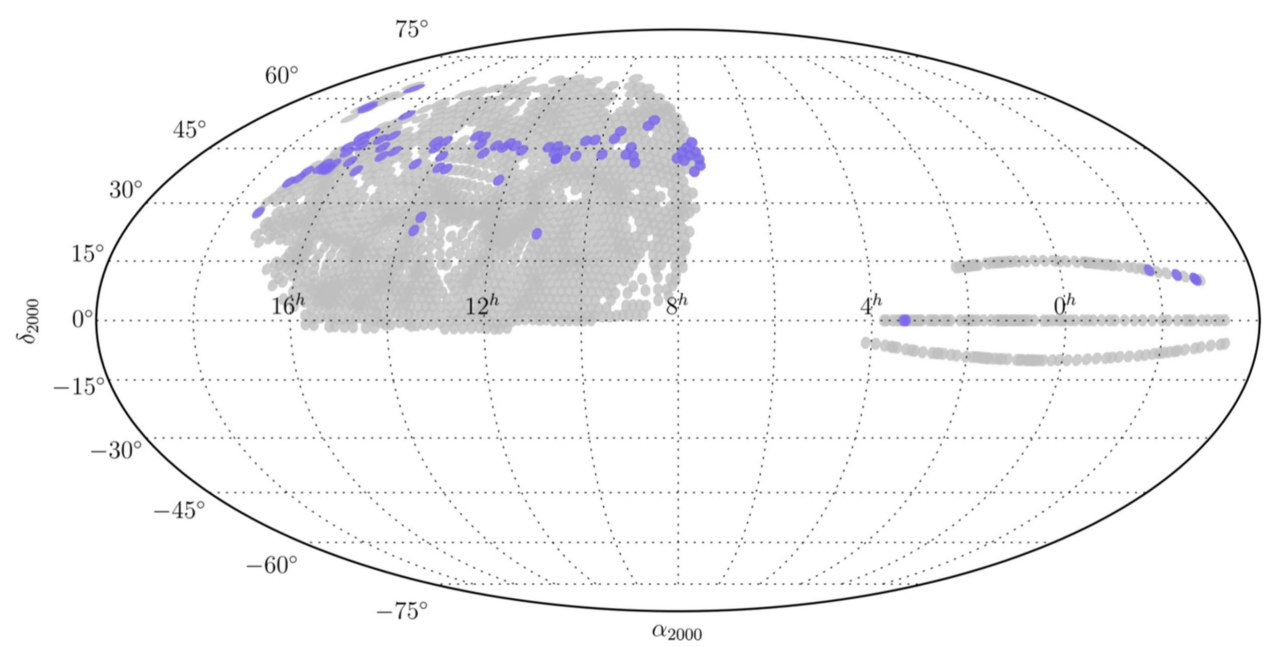

Figure 1. In gray are shown the locations in equatorial coordinates of all possible plates with MaNGA galaxies, each containing 17 galaxy targets. Because the MaNGA targets are selected to have SDSS photometry, this footprint corresponds to the Data Release 7 imaging data (Abazajian et al. 2009). Approximately 30\% of these will be observed in the full planned MaNGA survey. The purple shows the plates observed in the first year of MaNGA for which data cubes are released in this paper.

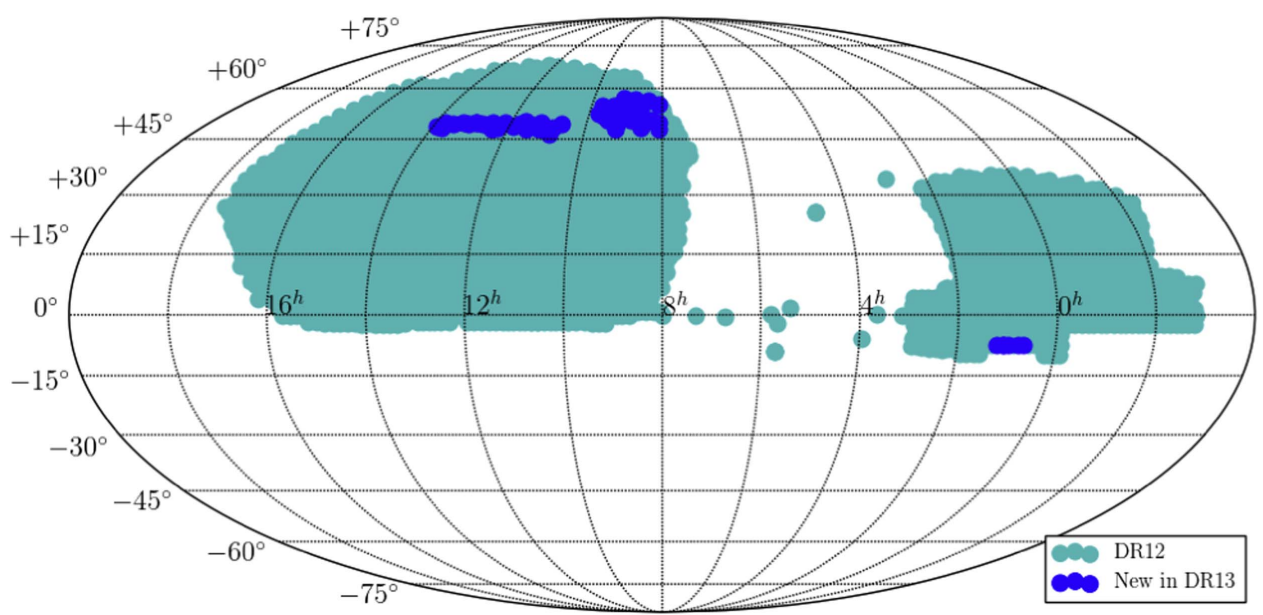

Figure 2. Coverage of DR13 data from BOSS and SEQUELS in equatorial coordinates. The blue areas show the locations in equatorial coordinates of the five new BOSS plates (SGC) and the 126 SEQUELS plates (NGC) released in DR13. The green represents the area covered by BOSS in DR12. The SEQUELS plates released in DR12 lie in the same region as the new ones in DR13, providing complete coverage over roughly $400 \mathrm{deg}^{2}$.

Links to all of these methods are provided at the SDSS web site (http://www.sdss.org/dr13/data_access). The data processing software for APOGEE, BOSS, and SEGUE are publicly available at http://www.sdss.org/dr13/software/ products. A set of tutorial examples for accessing SDSS data is provided at http://www.sdss.org/dr13/tutorials. All flat files are available for download at http://data.sdss.org/ datamodel/.

\section{Recalibration of Imaging Data}

DR13 includes a photometric recalibration of the SDSS imaging data. Finkbeiner et al. (2016) rederived the $g_{-}, r-, i$, and $z$-band zero-points using the PS1 photometric calibrations of Schlafly et al. (2012), as well as rederiving the flat fields in all five bands (including $u$ ). This effort improved the accuracy of the SDSS photometry previously hindered by a paucity of overlapping scans to perform ubercalibration across the entire SDSS sky and by suboptimal flat fields. The residual systematics, as measured by comparison with PS1 photometry and spectral energy distributions for stars with spectra, are reduced to $0.9 \%, 0.7 \%, 0.7 \%$ and $0.8 \%$ in the griz bands, respectively; several previously uncertain calibrations of specific runs are also now much better constrained. The resulting recalibrated imaging catalogs are the basis for the eBOSS and MaNGA targeting.

For the MaNGA target selection, we are using the NASASloan Atlas (NSA; Blanton et al. 2011), a reanalysis of the SDSS photometric data using sky subtraction and deblending better tuned for large galaxies. Relative to the originally distributed version of that catalog, we have used the new calibrations mentioned above, increased the redshift range up to $z=0.15$, and added an elliptical aperture Petrosian measurement of flux, which MaNGA targeting is based on. DR13 includes the NSA catalog (version V1_0_1) associated with this reanalysis as the nsatlas CAS table and as a file on the SAS. For the MaNGA galaxies released in DR13, we provide the actual images (referred to in MaNGA documentation as "preimaging") on the SAS as well.

Lang et al. (2016) reanalyzed data from the Wide-field Infrared Satellite Explorer (WISE; Wright et al. 2010) to use 


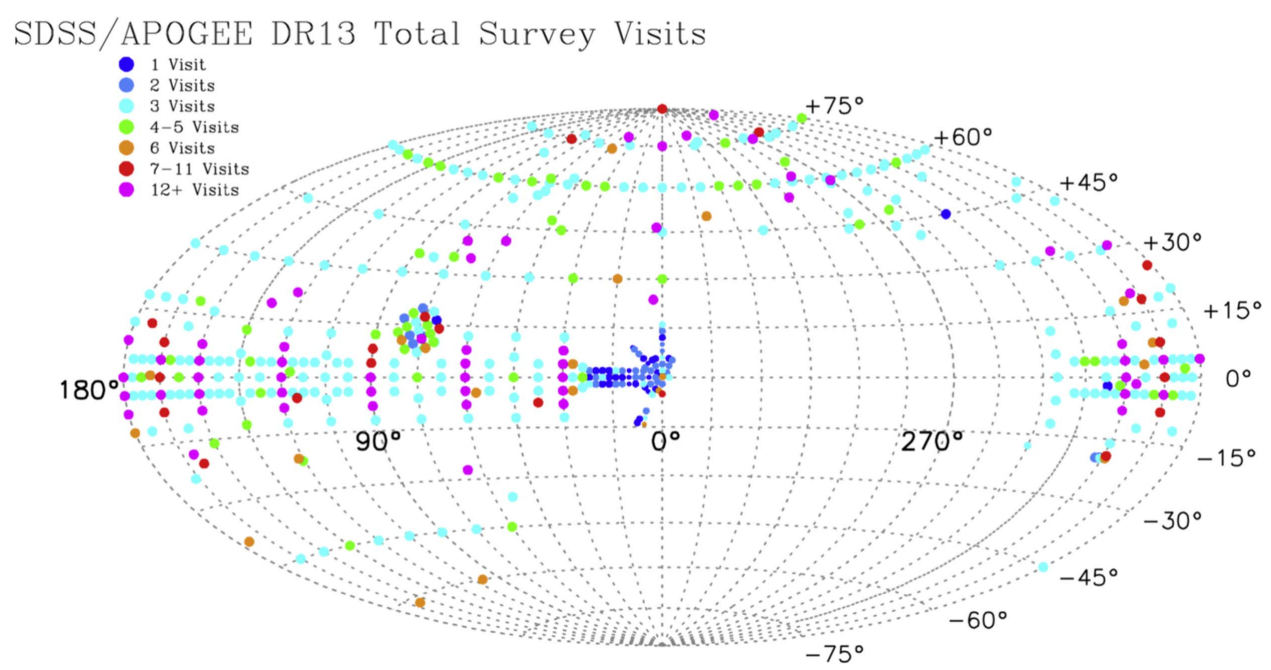

Figure 3. Coverage of APOGEE-2 DR13 data in Galactic coordinates; the raw data and its coverage are the same as in DR12, but they have been reprocessed through the latest reduction pipeline and ASPCAP versions. The color-coding denotes the number of visits to each field, as indicated in the legend.

for eBOSS targeting. They used positions and galaxy profile measurements from SDSS photometry as input structural models and constrained fluxes in the WISE $3.4 \mu \mathrm{m}$ and 4.6 $\mu \mathrm{m}$ bands. These results agree with the standard WISE reductions to within $0.03 \mathrm{mag}$ for high signal-to-noise ratio, isolated point sources in WISE. However, the new reductions provide flux measurements for low signal-to-noise ratio $(<5 \sigma)$ objects detected in the SDSS but not in WISE (over 200 million objects). Despite the fact that the objects are undetected, their flux measurements are nevertheless informative for target selection, in particular for distinguishing stars from quasars. This photometry is provided as a value-added catalog in the wiseForcedTarget CAS table and on the SAS as described in Table 2.

The Galactic extinction estimates published in the SDSS imaging tables (photoobj and related tables in the CAS) have been changed. The Galactic extinction still uses the Schlegel et al. (1998) models of dust absorption to estimate $E(B-V)$, but the Galactic extinction coefficients for each band have been updated as recommended by Schlafly \& Finkbeiner (2011). The extinction coefficients $R_{u}, R_{g}, R_{r}, R_{i}$, and $R_{z}$ are changed from the values used in BOSS (5.155, $3.793,2.751,2.086,1.479)$ to updated values $(4.239,3.303$, $2.285,1.698,1.263)$. The corresponding numbers for the WISE bands are $R_{W 1}=0.184$ for the WISE $3.4 \mu \mathrm{m}$ band and $R_{W 2}=0.113$ for the $4.6 \mu \mathrm{m}$ band (Fitzpatrick 1999).

\section{MaNGA: Integral Field Spectroscopic Data}

MaNGA is investigating the internal kinematics and composition of gas and stars in low-redshift $(z \leqslant 0.15)$ galaxies using fiber bundles to feed the BOSS spectrographs. Bundy et al. (2015) describe the high-level scientific goals, scope, and context of the survey in investigating galaxy formation, while Yan et al. (2016) give a detailed description of the survey design, execution, and data quality relevant to DR13. With nearly 1390 data cubes released, MaNGA's DR13 data products represent the largest public sample to date of galaxies observed with integral field spectroscopy. This data set signifies a valuable first step in MaNGA's goals to reveal the internal properties and dynamics of a statistically powerful sample of galaxies, which spans a broad range in stellar mass, local environment, morphology, and star formation history. Individual observations across the sample are of sufficient quality to characterize the spatially dependent composition of stars and gas, as well as their internal kinematics, thus providing important clues on growth and star formation fueling, the buildup of spheroidal components, the cessation of star formation, and the intertwined assembly history of galaxy subcomponents.

The survey was made possible through an instrumentation initiative in SDSS-IV to develop a reliable and efficient way of bundling 1423 optical fibers into tightly packed arrays that constitute MaNGA's IFUs (Drory et al. 2015). For each pointing, MaNGA observes 17 science targets with IFUs ranging from 19 to 127 fibers (with diameters of $12^{\prime \prime}-32^{\prime \prime}$ ). The IFU size distribution was optimized in concert with the sample design (Wake et al. 2017) that targets SDSS-I/II main sample galaxies at a median redshift of 0.03 to obtain in $6 \mathrm{yr}$ a sample of $10^{4}$ galaxies with uniform radial coverage and a roughly flat distribution in $\log M_{*}$ limited to $M_{*}>10^{9} M_{\odot}$. Careful attention was paid to optimizing hardware and an observing strategy that ensures high-quality imaging spectroscopy (Law et al. 2015) and to surface photometric flux calibration with a precision better than $5 \%$ across most of the wavelength range, 3622-10354 $\AA$ (Yan et al. 2016). As described in these papers, salient aspects included protocols for constraining hour angles of observations to limit differential atmospheric diffraction, dithering exposures to avoid gaps in the coverage of the targets because of space between the fibers, and special calibration minibundles to ensure reliable absolute and relative flux calibration. An automated pipeline delivers sky-subtracted, flux-calibrated row-stacked spectra (RSS) and data cubes for all sources (Law et al. 2016).

\subsection{MaNGA DR13 Main Galaxy Sample}

At the completion of SDSS-IV, the MaNGA survey's main galaxy sample will include $\sim 10^{4}$ galaxies with $M_{*}>10^{9} M_{\odot}$ and a roughly flat stellar mass distribution. DR13's 1390 galaxy data cubes, corresponding to 1351 unique galaxies, makes it the largest public sample of galaxies with IFU spectroscopy. MaNGA's main galaxy sample consists of three 
Table 2

Value-added Catalogs New in DR13

\begin{tabular}{|c|c|c|}
\hline Catalog Description & References & http://data.sdss.org/sas/dr13/ \\
\hline SPIDERS cluster demonstration sample catalog & Clerc et al. (2016) & eboss/spiders/analysis/ \\
\hline SPIDERS AGN target selection catalog & Dwelly et al. 2017 & eboss/spiders/target/ \\
\hline SPIDERS cluster target selection catalog & Clerc et al. (2016) & eboss/spiders/target/ \\
\hline WISE forced photometry & Lang et al. (2016) & eboss/photoObj/external/WISEForcedTarget/301/ \\
\hline Composite spectra of emission-line galaxies & Zhu et al. (2015) & eboss/elg/composite/v1_0/ \\
\hline ELG Fisher selection catalog & Delubac et al. (2017) & eboss/target/elg/fisher-selection/ \\
\hline Redmonster redshift and spectral classification catalog & Hutchinson et al. (2016) & eboss/spectro/redux/redmonster/v5_9_0/v1_0_1/ \\
\hline QSO variability & Palanque-Delabrouille et al. (2016) & eboss/qso/variability/ \\
\hline APOGEE DR13 red-clump catalog & Bovy et al. (2014) & apogee/vac/apogee-rc/cat/ \\
\hline
\end{tabular}

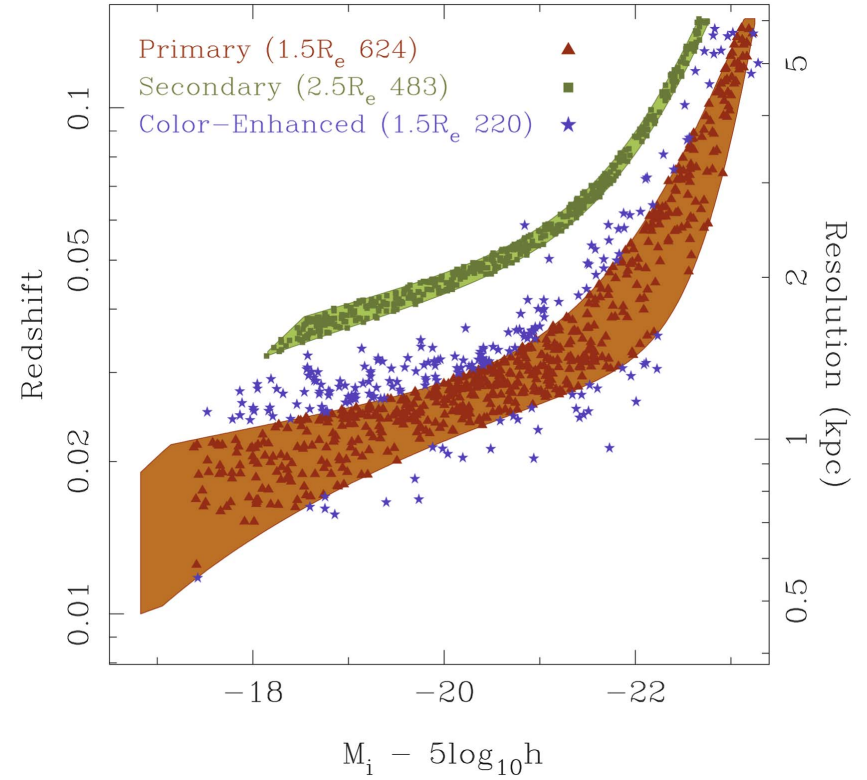

Figure 4. Principal selection cuts for the main MaNGA samples, where $h=H_{0} / 100 \mathrm{~km} \mathrm{~s}^{-1}$. The colored bands show the selection cuts for the Primary (orange) and Secondary (green) samples, illustrating the $M_{i}$ dependence of the redshift limits. More luminous and hence typically larger galaxies are selected at higher redshift than less luminous galaxies, ensuring that the angular size $\left(1.5 R_{e}\right.$ or $\left.2.5 R_{e}\right)$ distribution is roughly independent of luminosity. The volume sampled also increases as the luminosity increases in such a way as to ensure a constant number density of galaxies at all luminosities. The points show the positions in this plane for the MaNGA galaxies in DR13, for the Primary (red triangles), Secondary (green squares), and Color-enhanced samples (blue stars), although the colorenhanced selection also depends on NUV-icolor (see text for details). The numbers in the legend give the total number of observations of galaxies in each class, including repeat observations. The right-hand $y$-axis gives an indication of the physical size of the mean spatial resolution element of the MaNGA data.

major parts: Primary sample, Secondary sample, and the Colorenhanced supplement.

The Primary sample and the Secondary sample are selected from two luminosity-dependent redshift bands, as shown in Figure 4. The selection for each sample is volume limited at each absolute $i$-band magnitude. The shape of the redshift bands is motivated by MaNGA's science requirements of having a uniform spatial coverage in units of a galaxy's effective radius $R_{e}$ and having a roughly flat stellar mass distribution (Yan et al. 2016; Wake et al. 2017). Figure 5 shows the distribution of the MaNGA DR13 galaxies in the stellar mass versus dark matter halo mass plane. Because more massive galaxies are on average larger, we observe them at a larger distance than low-mass galaxies. We chose the redshift bands so that the great majority of the Primary (Secondary) sample is covered by our fiber bundles to $1.5 R_{e}\left(2.5 R_{e}\right)$ along their major axes. This has the commensurate effect of changing the physical resolution systematically as a function of stellar mass, as illustrated in Figure 4. Potential deleterious effects of this change in sampling are addressed by an ancillary program, described below.

We also designed a color-enhanced supplement, as a supplement to the Primary sample, to enhance the sampling of galaxies with rare color-magnitude combinations, such as low-mass red galaxies, high-mass blue galaxies, and green valley galaxies. This is achieved by extending the redshift limits around the Primary sample redshift band for each underpopulated region in color-magnitude space.

The combination of the Primary sample and the Colorenhanced supplement is referred to as the Primary+ sample. We provide in our data release the redshift limits over which each galaxy is selected. This permits a correction to the sample using $1 / V_{\max }$ weight to reconstruct a volume-limited representation of the galaxy population, provided that there is negligible galaxy evolution over this limited redshift range. More details of how we arrived at this selection can be found in Yan et al. (2016) and Wake et al. (2017). Wake et al. (2017) provide the details of how to properly weight the sample to reconstruct a volume-limited representation.

Among the 1351 unique galaxies released in DR13, there are 600 Primary sample galaxies, 473 Secondary sample galaxies, and 216 Color-enhanced supplement galaxies. There are 62 galaxies that do not belong to any of the above. Some of these are ancillary program targets (see below), some are filler objects on plates with spare bundles, and others are galaxies selected using older, obsolete versions of the selection and observed on early plates. For most statistical analyses, these 62 galaxies should be excluded.

Which sample a given target galaxy belongs to is given by the MANGA_TARGET1 bitmask (or mngtarg1 in the "drpall" file; see Section 5.2). Primary sample galaxies have bit 10 set to 1, Secondary sample galaxies have bit 11 set to 1 , and Colorenhanced supplement galaxies have bit 12 set to 1 . Bits 1-9 are for obsolete selections and should be ignored.

\subsubsection{MaNGA Galaxy Ancillary Programs}

Roughly $5 \%$ of the IFUs are assigned to ancillary science programs defined by and allocated through internal competition and review. This assignment takes advantage of sky regions with a low density of galaxies defined by our main survey 

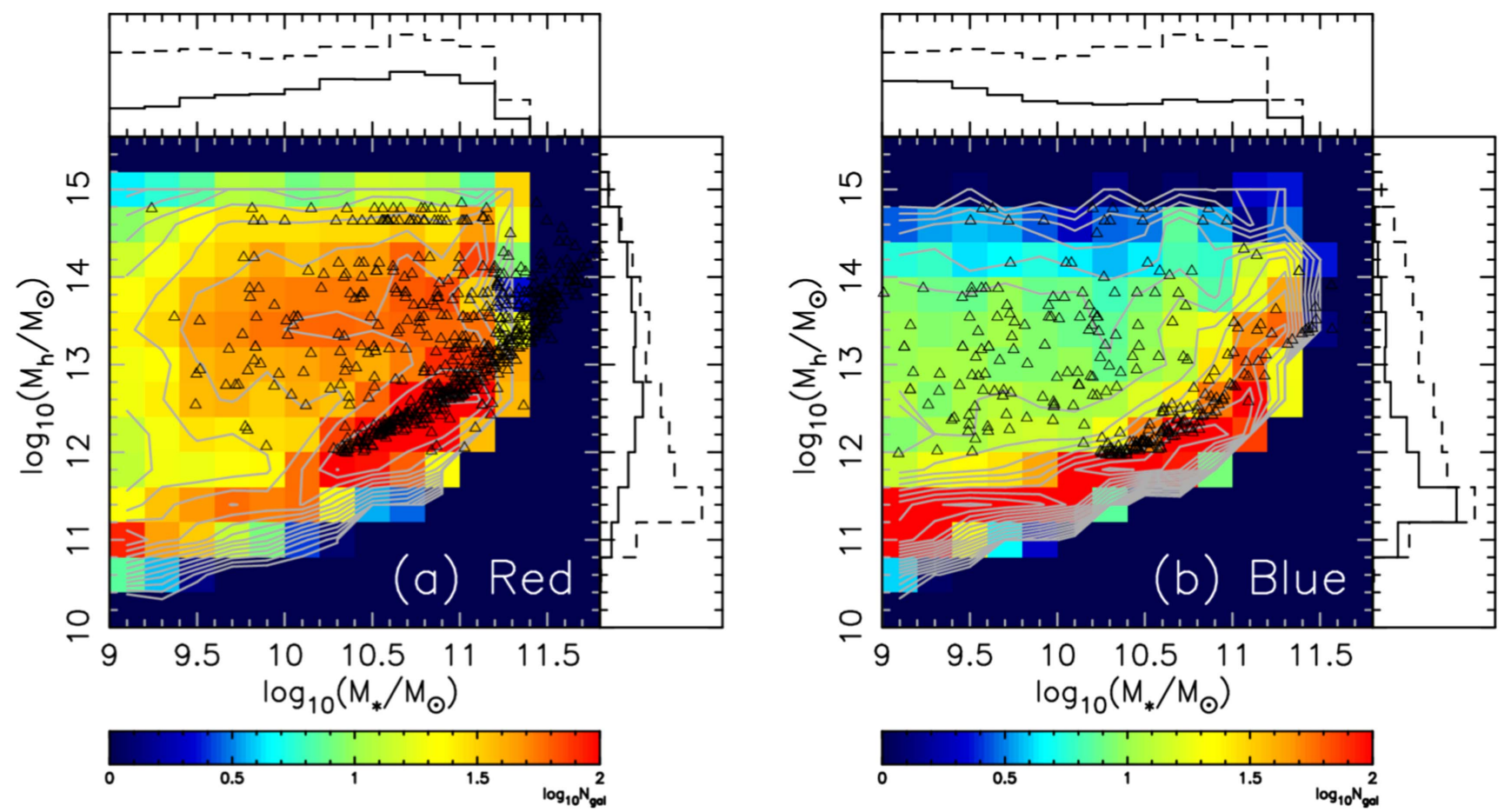

Figure 5. Location of MaNGA galaxies (black triangles) in this data release area in the plane of stellar mass $\left(M_{*}, x\right.$-axis) vs. dark matter halo mass $\left(M_{h}, y\right.$-axis). The two panels show red and blue galaxies separately, which are divided by a single color cut at $g-r=0.7$. Stellar masses are taken from the NASA-Sloan Atlas, and halo masses from SDSS /DR7, using the method of Yang et al. (2007). Plotted as a color map in the background are the number of MaNGA galaxies predicted for a 6 yr full survey based on mock catalogs informed by the semianalytic model of Guo et al. (2011) and constructed as in Li et al. (2006), which are the same as Figure 3 of Bundy et al. (2015). Normalized histograms show 1D marginalized $M_{*}$ distributions (top axes) and $M_{h}$ distributions (right axes), with dashed lines for the full Primary sample and solid lines for the red (left) and blue (right) populations.

criteria (above) or where certain rare classes of objects, possibly outside our selection cuts, are of sufficiently high science value to re-allocate IFUs from the main program. Such high-value targets sometimes come from the main sample, but the ancillary science goals require a different bundle size, a slightly different center position, or higher prioritization over the random selection among the main sample galaxies. There are also science cases where using observing strategies different from standard MaNGA observations is required. These lead to dedicated plates.

We solicited ancillary proposals in all these categories during the first year of survey observations, and they started to be included in plate design halfway through this year. Consequently, the ancillary fraction in DR13 is smaller than 5\%. Some ancillary programs have tens of targets approved, but only a few got observed during the first year, while some have no observations during this period. More targets for these programs will be observed in the future. To identify ancillary targets, one should use the MANGA_TARGET3 bitmask (or mngtarg3 in the drpall file). All ancillary targets have MANGA_TARGET3 greater than zero. The bitmask names and values for the programs with data for at least one galaxy in DR13 are given in Table 3. Additional information on the scientific justification and targeting for each ancillary program can be found at http://www.sdss.org/dr13/ manga/manga-target-selection/ancillary-targets/. Here we provide some highlights and the corresponding bitnames:

1. Luminous AGNs: this program increases the number of host galaxies of the most luminous active galactic nuclei (AGNs). The first source of targets is the BAT 70-month Hard X-ray catalog (Baumgartner et al. 2013). These have the bitname AGN_BAT. To increase the sample size further, we used the [O III]-selected catalog of Mullaney et al. (2013) (bitname AGN_OIII). To match the distribution of bolometric luminosities between the samples, we selected five [O III] objects at comparable $L_{\text {bol }}$ to each BAT object, within a redshift range of 0.01-0.08. The bolometric corrections are from Shao et al. (2013) and Vasudevan \& Fabian (2009).

2. Edge-on Starbursts: we will use edge-on starbursts to study the morphology and ionization state of large-scale outflows. To identify good targets, the specific star formation rate (sSFR) and inclination of every object in the baseline MaNGA targeting catalog were calculated. The sSFR was determined using WISE photometry from Lang et al. (2016) and the calibration between the W4 filter and $22 \mu \mathrm{m}$ emission in Jarrett et al. (2013). We then use a calibration from Cluver et al. (2014) to derive the sSFR. The axis ratio SERSIC_BA in the targeting catalog was used to derive the inclination. All targets in this program have log $\mathrm{sSFR}>-8.75$ and inclination $>75$ deg. The four galaxies in DR13 that have these properties but were not included in the main galaxy target sample have bitname EDGE_ON_WINDS.

3. Close Pairs and Mergers: interactions and mergers can play a key role in galaxy evolution, and therefore an ancillary program was accepted that either slightly adjusted the field centers for some targets already included in the main galaxy sample or placed new IFUs on galaxies. Close pairs are defined as galaxies in the NSA catalog or the SDSS group catalog of Yang et al. (2007) and X. Yang (2017, private communication) with projected separation $<50 \mathrm{kpc} h^{-1}$ and line-of-sight velocity $(d V)<500 \mathrm{~km} \mathrm{~s}^{-1}$, if both redshifts are 


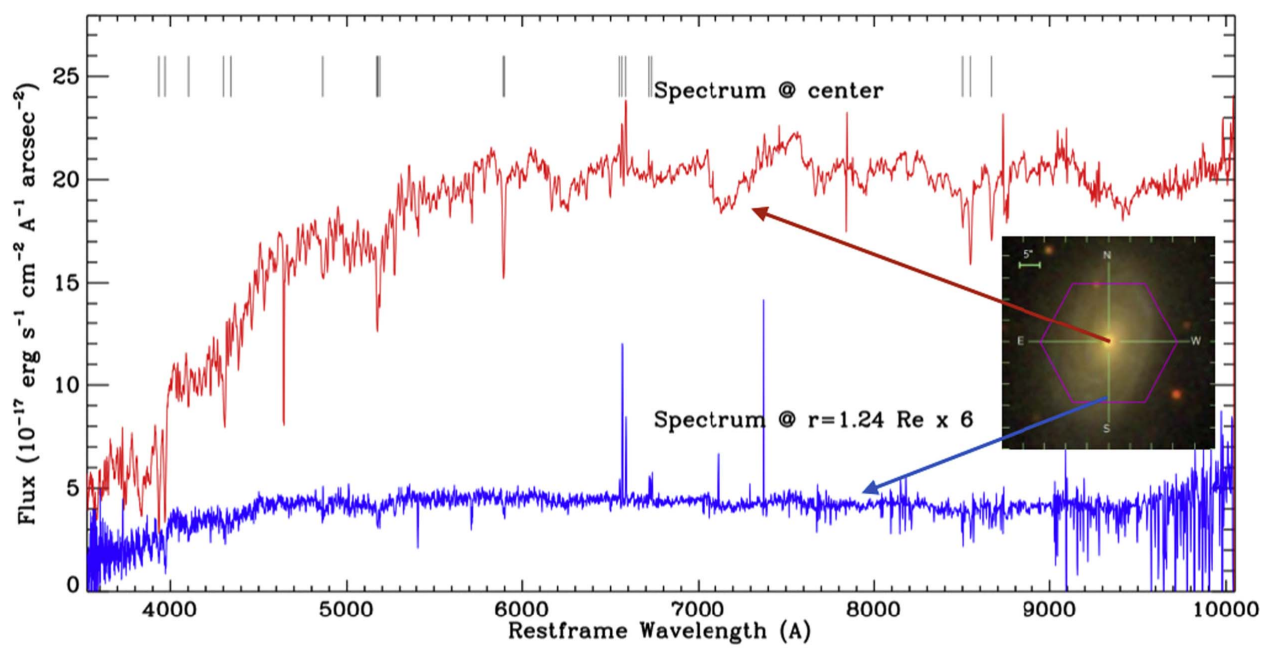

Figure 6. Example spectra from a typical MaNGA data cube, adapted from Yan et al. (2016). The inset shows the SDSS color image with the hexagonal IFU footprint overlaid. The top spectrum is from the central spaxel; the bottom spectrum is from a spaxel $1.2 R_{e}$ from the center and is multiplied by a factor of 6 for easier comparison with the central spectrum. The differences in the stellar and gas components between the two regions can clearly be seen, as well as the large number of diagnostic features to understand the star formation history and the physical conditions of the gas.

available. If the bitname is PAIR_ENLARGE, then to get the full pair required a larger IFU than the one originally scheduled by the targeting algorithm (Wake et al. 2017). If the bitname is PAIR_RECENTER, this means that the original assigned MaNGA IFU is sufficiently large but requires re-centering. In addition to these already-planned galaxies, two sources of new objects were used. The one galaxy in DR13 with PAIR_SIM comes from the Galaxy Zoo Mergers Sample (Holincheck et al. 2016). A critical sample comes from the ancillary program that requests that each galaxy be assigned a separate IFU PAIR_2IFU. Only four pairs of interacting galaxies are serendipitously targeted in the main MaNGA galaxy sample with separate IFUs, and this sample will compensate for the strong bias in the single IFU sample toward close separations or higher redshifts.

4. Massive Nearby Galaxies: because the largest MaNGA IFU covers $32^{\prime \prime}$, more luminous, larger galaxies observed to the same effective radius have poorer spatial resolution. The one MASSIVE ancillary target in DR13 is part of a program to obtain a sample of nearby large galaxies with spatial resolution better than $3 \mathrm{kpc}$ and similar to the faintest galaxies in the MaNGA Primary sample, at the cost of spatial extent.

5. Milky Way Analogs: Licquia et al. (2015) defined a sample of Milky Way analogs based on $M_{*}$, SFR, absolute magnitudes, and colors. MaNGA is including some of these analogs in the main galaxy catalog, but they are slightly biased or deficient in certain regions of parameter space. Galaxies with the bitname MWA are drawn from the Licquia et al. (2015) catalog to provide galaxies in those underrepresented parts of parameter space.

6. Dwarf Galaxies in MaNGA: the MaNGA main galaxy sample has galaxies with $M_{*}>10^{9} M_{\odot}$, but dwarf galaxies are the most numerous galaxies in the universe. This ancillary program provides two dwarf galaxies with MaNGA observations in DR13, the first observations of a larger sample expected by the end of the survey covering a range of environments. These galaxies are indicated by the bitname DWARF and are drawn from the Geha et al. (2012) galaxy catalog with stellar masses $<10^{9} M_{\odot}$.
7. Brightest Cluster Galaxies: the brightest cluster galaxies (BGCs) targeted here are brighter and in more massive halos than BCGs already in the MaNGA main sample and have the bitname BCG. We base our target selection on the updated Yang et al. (2007) cluster catalog, created from the SDSS DR7 NYU VAGC, an update of the DR4 version of Blanton et al. (2005).

8. MaNGA Resolved Stellar Populations: the ancillary program targets NGC 4163, a nearby dwarf galaxy with existing HST imaging and high-quality color-magnitude diagrams selected from the ACS Nearby Galaxy Survey (Dalcanton et al. 2009). This galaxy is flagged by the bitname ANGST.

\subsection{MaNGA Data Products: Individual Fiber Spectra and 3D Data Cubes}

In DR13, MaNGA is releasing both raw data (in the form of individual CCD frames) and reduced data produced by version 1_5_4 of the MaNGA Data Reduction Pipeline (DRP). Figure 6 illustrates the quality of the spectra from this pipeline. The MaNGA observing strategy is described by Law et al. (2015), and the flux calibration by Yan et al. (2016). Details of the MaNGA DRP, data products, and data quality are given by Law et al. (2016, hereafter L16). All MaNGA data are in the form of multi-extension FITS files.

The DRP data products consist of intermediate reduced data (sky-subtracted, flux-calibrated fiber spectra with red and blue data combined for individual exposures of a plate) and finalstage data products (summary row-stacked spectra and data cubes) for each target galaxy. The summary row-stacked spectra (RSS files) are 2D arrays provided for each galaxy in which each row corresponds to a single-fiber spectrum. For a 127-fiber IFU with nine exposures, there are thus $127 \times 9=1143$ rows in the RSS file. These RSS files have additional extensions giving astrometric information about the wavelength-dependent locations of each fiber on the sky.

The 3D data cubes (axes R.A., decl., wavelength) are created by combining the individual spectra for a given galaxy together onto a regularized 0 ". 5 grid (see L16 for more detail). Both data cubes and RSS files are provided in a version with a $\log$ 
wavelength scale, which is the standard extraction and is relatively smooth in velocity space, and in a version with a linear wavelength scale, created directly from the native pixel solution rather than by resampling the log-scaled spectra resampling. Each MaNGA data cube has associated extensions corresponding to the estimated inverse variance per pixel and a bad-pixel mask containing information about the quality of a given pixel within the cube (depth of coverage, bad values, presence of foreground stars, etc.). Additional extensions provide information about the instrumental spectral resolution, individual exposures that went into the composite data cube, reconstructed griz broadband images created from the IFU spectra, and estimates of the griz reconstructed point-spread function (PSF).

The objects observed by MaNGA for which data cubes have been produced are summarized in the "drpall" file, a FITS binary table with one entry per object (including both galaxies and spectrophotometric standard stars observed with sevenfiber IFUs to calibrate the MaNGA data). This drpall file lists the name, coordinates, targeting information (e.g., redshift as given by the NASA-Sloan Atlas), reduction quality, and other quantities of interest to allow users to identify galaxy targets of interest. We note that MaNGA adopts two naming schemes. The first, termed "manga-id," is an identifier unique to a given astronomical object (e.g., 1-266039). The second, the "plateifu," uniquely identifies a given observation by concatenating the plate id with the IFU number (e.g., 7443-12701 identifies the first 127 -fiber IFU on plate 7443). Since some galaxies are observed more than once on different plates, a given manga-id can sometimes correspond to more than one plate-ifu.

The manga-id consists of two parts separated by a hyphen. The first part is the id of the parent catalog from which a target was selected. The second part is the position within that catalog. For most galaxy targets the catalog id is 1 , which refers to the NSA. For a small number of the early targets the catalog id is 12 and refers to an earlier version of the NSA (v1b_0_2). All galaxies from this earlier version of the NSA are also in the final version, and so we release photometry, etc., for those targets from the final version of the NSA (v1_0_1), which is included in the data release. Other catalogs referred to in the first part of the manga-id are for SDSS standard stars.

The full data model for all MaNGA DRP data products can be found online at http://www.sdss.org/dr13/manga/mangadata/data-model/ and is also given in Appendix B of L16.

\subsection{Retrieving MaNGA Data}

The raw data, reduced data, RSS, and 3D data cubes for 1351 MaNGA galaxies are provided in DR13. From these data products, maps of line ratios, spectroscopic indices, and kinematics can be made using standard software. Because the first step in using the MaNGA data for science is to retrieve the spectra, we detail here and on the SDSS web site ${ }^{131}$ how to access the MaNGA spectra.

\subsubsection{Reduced Data Products}

MaNGA DR13 reduced data products are stored on the Science Archive Server at http://data.sdss.org/sas/dr13/ manga/spectro/redux/v1_5_4/. The top-level directory contains the summary drpall FITS table and subdirectories for each

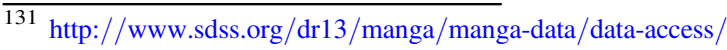

plate. Inside each plate directory there are subdirectories for each MJD on which the plate was observed, containing intermediate (exposure level) data products. The "stack" subdirectory within each plate directory contains the final RSS and cube files for each MaNGA galaxy, sorted according to their plate-ifu identifiers. Note that the ifu identifier in the file names indicates the size of the IFU; everything in the 127 series (e.g., 12701) is a 127-fiber bundle, etc. The 700 series ifus (e.g., 701) are the 12 spectrophotometric seven-fiber minibundles that target stars on each plate.

These are the ways of getting at the data in DR13:

1. Direct html browsing of the SAS at the above link. The file drpall-v1_5_4.fits can be downloaded through the web browser and queried using any program able to read FITS binary tables. Once a set of galaxies of interest has been identified, individual data cubes, summary RSS files, intermediate data products, etc., can be downloaded by browsing through the web directory tree.

2. Large downloads of many DRP data products can be automated using rsync access to the SAS. For instance, to download all MaNGA data cubes with a logarithmic wavelength format: \rsync-aLrvz-include"*/ "-include"manga*LOGCUBE. fits.gz"-exclude"*"rsync:// https://data.sdss.org/sas/dr13/ manga/spectro/redux/v1_5_4/.

3. The MaNGA drpall file can also be queried online using the SDSS CASJobs system at http://skyserver.sdss.org/ casjobs. While this can be useful for identifying MaNGA observations of interest, CASJobs does not contain links to the MaNGA data cubes, and another method must be used to download the data themselves.

4. The SDSS SkyServer Explore tool at http://skyserver. sdss.org/dr13/en/tools/explore/ will display basic information about MaNGA observations in DR13 that fall within a given cone search on the sky. The relevant explore pages also provide direct links to the FITS data cubes on the SAS.

\subsubsection{Raw Data}

All MaNGA data taken in the first year of SDSS-IV observations are part of Data Release 13, including data from special ancillary plates and co-observing during APOGEE-2 time that are not part of the DRP results. The raw data are stored on the SAS in the directory http://data.sdss.org/sas/ $\mathrm{dr} 13 / \mathrm{manga} /$ spectro/data/ in subdirectories based on the MJD when the data were taken. The mangacore directory ${ }^{132}$ contains the information needed to figure out the R.A. and decl. positions of fibers on plates, the dithered MJDs to be combined to make the final spectrum in apocomplete directory, and information on the calibration files. L16 and the http://www. sdss.org/dr13/manga/manga-data/metadata/ web site contain the relevant information about the file formats and the use of the calibration files to get to the fully reduced spectra. Because these files are prepared for internal use, they retain many old features that should be ignored, such as the names assigned to targeting bits, which still retain the names from SDSS-I.

\footnotetext{
132 http://svn.sdss.org/public/repo/manga/mangacore/tags/v1_2_3/
} 
Table 3

Summary of MaNGA Ancillary Programs with Data in DR13

\begin{tabular}{|c|c|c|c|c|c|}
\hline Ancillary Program & \# of Targets in DR13 ${ }^{\mathrm{a}}$ & \# of Total Targets ${ }^{\mathrm{a}, \mathrm{b}}$ & PITNAME & Binary Digit & $\overline{\text { Value }}$ \\
\hline \multirow[t]{2}{*}{ Luminous AGNs } & 1 & 267 & AGN_BAT & 1 & 2 \\
\hline & 4 & & AGN_OIII & 2 & \\
\hline Edge-on star-forming galaxies & 4 & 166 & EDGE_ON_WINDS & 6 & 64 \\
\hline & 10 & & PAIR_RECENTER & 8 & 256 \\
\hline & 1 & & PAIR_SIM & 9 & 512 \\
\hline & 1 & & PAIR_2IFU & 10 & 1024 \\
\hline Dwarf galaxies in MaNGA & 2 & 247 & DWARF & 14 & 16384 \\
\hline Brightest cluster galaxies & 2 & 378 & BCG & 17 & 131072 \\
\hline MaNGA resolved stellar populations & 1 & 4 & ANGST & 18 & 262144 \\
\hline
\end{tabular}

Notes.

${ }^{\text {a }}$ An individual galaxy can be targeted by more than one ancillary program.

${ }^{b}$ Number for each ancillary program refers to all targets in that program, regardless of bitname.

\subsection{Notes on Using MaNGA Data}

There are several important caveats to keep in mind when working with MaNGA data. In this discussion we treat only the MaNGA data cubes. Summary RSS, intermediate, and even raw data products present some statistical advantages to the data cubes, in particular reduced covariance between adjacent data points and greater ease of forward modeling, but are substantially harder to use.

First and foremost, each MaNGA data cube has a FITS header keyword DRP3QUAL indicating the quality of the reduction. $1 \%-2 \%$ of the data cubes are flagged as significantly problematic for various reasons, ranging from poor focus to flux calibration problems. Table B13 in L16 lists the bits that can be set with this flag that describe why the end product was deemed unsatisfactory. Galaxies for which the CRITICAL quality bit $(=30)$ is set in DRP3QUAL should be treated with extreme caution. While there may be some spaxels in that data cube that are acceptable, in general the CRITICAL bit indicates widespread problems with the data reduction. Each data cube also has an associated MASK cube describing the quality of individual spaxels in the data cube. This MASK extension can be used to identify areas of no coverage outside the fiber bundle footprint, ${ }^{133}$ low coverage near the edge of the dithered footprint, problematic areas due to detector artifacts, regions known to contain bright Milky Way foreground stars, etc. A simple summary DONOTUSE bit is of particular importance, indicating elements that should be masked out for scientific analyses.

Since the MaNGA data cubes adopt a 0."5 sampling size (chosen based on Fourier analysis in optimal observing conditions to not truncate the high- $k$ modes of the observational PSF), while individual fibers have a $2^{\prime \prime}$ diameter footprint, there is significant covariance between adjacent MaNGA spaxels that must be taken into account whenever combining spectra. A simplified method for doing this is discussed in Section 9.3 of L16. The typical reconstructed PSF of the MaNGA data cubes has FWHM of 2!"5.

\footnotetext{
133 The fiber footprint is a hexagon, but the standard FITS image data structure is based around rectangular arrays. There must therefore be blank areas around the live IFU footprint in order to inscribe the hexagon inside a bounding rectangle.
}

As discussed by L16, the instrumental line-spread function (LSF) in the wavelength direction reported by the various extensions within the MaNGA DR13 data products is underestimated by about $10 \% \pm 2 \%$. This correction is comparable to the errors in the reported resolution of the BOSS spectrographs seen in single-fiber work (e.g., Shu et al. 2012; Palanque-Delabrouille et al. 2013) Although this makes little difference when determining the astrophysical width of broad spectral lines, it is important to account for when attempting to subtract the instrumental resolution from barely resolved lines. There are two effects that combine to produce this overestimate. The first is that the impact of the wavelength rectification on the effective spectral resolution was not accounted for when combining spectra from blue and red cameras. The second is that the Gaussian width of the LSF reported by the DRP is strictly the width of a pre-pixelization Gaussian, while most end-user analysis routines adopt postpixelization Gaussians instead (i.e., the difference between integrating a Gaussian profile over the pixel boundaries and evaluating a Gaussian at the pixel midpoint). This will be treated more completely in a future data release; in the meantime a $10 \%$ correction to the instrumental LSF quoted by the MaNGA data products appears to be a reasonable correction factor if using post-pixelization analysis routines. However, because this correction factor itself is uncertain, derived astrophysical line widths substantially below the instrumental resolution should be viewed to have unreliable accuracy at this time. A full discussion of issues to consider is available at http://www.sdss.org/dr13/manga/manga-caveats/.

\subsection{Highlights of MaNGA Science with DR13 Data}

The MaNGA survey has produced a number of scientific results based on early data, indicating the breadth of research possible with the MaNGA data. Here we briefly summarize the results of papers completed within the SDSS-IV collaboration using MaNGA data on spatially resolved gas physics, stellar population properties, and stellar and gas kinematics. These MaNGA papers serve as a guide to prospective users of SDSSIV data in these specific science areas. The larger context for this science is provided to a small degree in the Introduction, and the papers here also provide citations to the extensive 
literature on each topic, to which we refer the interested reader for additional context. Citations to the science in each area should refer to the original papers, whether MaNGA-based or not, rather than this brief executive summary.

\subsubsection{Gas Physics}

The spatially resolved emission-line measurements have been used to understand the physical conditions of the ionized gas in galaxies. Cheung et al. (2016a) identified AGN winds as a surprisingly common occurrence in normal, quiescent galaxies, suggesting these winds as potentially critical in suppressing star formation. These winds may help address the question of how star formation remains suppressed in earlytype galaxies. Cheung et al. (2016a) report bisymmetric emission features co-aligned with strong ionized-gas velocity gradients in a galaxy from which they infer the presence of centrally driven winds in typical quiescent galaxies that host low-luminosity active nuclei. These galaxies account for as much as $10 \%$ of the quiescent population with masses around $2 \times 10^{10} M_{\odot}$. They calculate that the energy input from the galaxies' low-level active supermassive black hole is capable of driving the observed wind, which contains sufficient mechanical energy to heat ambient, cooler gas (also detected) and thereby suppress star formation.

The broader nature of ionized gas in early types has also been the subject of papers by Belfiore et al. (2016) and Belfiore et al. (2017) following up on the analysis of a small sample observed with the MaNGA prototype instrument in Belfiore et al. (2015). By using spatially resolved maps of nebular diagnostics and stellar population ages, this work has added substantial support to the notion that evolved stellar populations provide the ionization source for a galaxy class that arguably should be renamed from LINER (Low Ionization Nuclear Emission Region) galaxies to LIER galaxies. LIERs, it turns out, are ubiquitous in both quiescent galaxies and the central regions of galaxies where star formation takes place at larger radii. The studies of Belfiore et al. (2016) and Belfiore et al. (2017) have put the occurrence of the LIER phenomenon into a physically relevant framework that can be directly tied to the diversity of the galaxy population as a whole. Specifically, they identify two classes of galaxies as extended LIER (eLIER) and central LIER (cLIER), respectively, and study their kinematics and stellar population properties. cLIERs turn out to be mostly late-type galaxies located around the green valley, while eLIERs are morphologically early types and are indistinguishable from passive galaxies devoid of line emission in terms of their stellar populations, morphology, and central stellar velocity dispersion.

The widespread ionization state of LIER gas might originally manifest as the diffuse intergalactic gas (DIG) that is intermixed with star-forming regions. Zhang et al. (2017) studied galactic DIG emission and demonstrate how DIG in star-forming galaxies impacts the measurements of emissionline ratios at the spatial resolution of MaNGA, hence the interpretation of diagnostic diagrams and the gas-phase metallicity measurements. They quantify for the MaNGA data how the contamination by DIG moves $\mathrm{H}$ II regions toward a composite of LIER-like regions. DIG significantly biases measurements of gas metallicity and metallicity gradients because at different surface brightness, line ratios and line ratio gradients can differ systematically.
A careful treatment of gas-phase metallicities inferred from spectral maps of galaxies has suggested a key role for the dependence of metallicity on local stellar mass surface density. Barrera-Ballesteros et al. (2016) present the stellar surface mass density versus gas metallicity relation for more than 500,000 spatially resolved star-forming regions from a sample of 653 disk galaxies. These galaxies span a larger range in mass than in previous samples where the correlations were first discovered. They confirm a tight relation between these local properties, with higher metallicities as the surface density increases. They find that even over this larger mass range this local relationship can simultaneously reproduce two wellknown properties of disk galaxies: their global massmetallicity relationship and their radial metallicity gradients. Their results support the idea that in the present-day universe local properties play a key role in determining the gas-phase metallicity in typical disk galaxies.

However, Cheung et al. (2016b) have found a galaxy in the middle of a gas accretion event, providing a detailed look at what appears to be a relatively rare occurrence in the nearby universe of this mode of galaxy growth. They present serendipitous observations of a large, asymmetric $\mathrm{H} \alpha$ complex that extends $\sim 8^{\prime \prime}(\sim 6.3 \mathrm{kpc}$ ) beyond the effective radius of a dusty, starbursting galaxy. This $\mathrm{H} \alpha$ extension is approximately three times the effective radius of the host galaxy and displays a tail-like morphology. This is suggestive of an inflow, which is consistent with its relatively lower gas-phase metallicities and its irregular gaseous kinematics.

\subsubsection{Stellar Populations}

Spatially resolved stellar population properties and stellar growth histories have been analyzed, following the analysis of a small sample observed with the MaNGA prototype instrument by Wilkinson et al. (2015) and Li et al. (2015). MaNGA has explored the role of environment in shaping the radial distribution of stellar ages and metallicities, with particular attention given to the potential measurement systematics. Using different spectral fitting techniques and complementary environmental metrics, both Goddard et al. (2017b) and Zheng et al. (2017) conclude that any environmental signal in the average shape of gradients is weak at best, with no obvious trends emerging in the initial MaNGA data.

Goddard et al. (2017a) studied the internal gradients of the stellar population age and metallicity within $1.5 R_{e}$ obtained from full spectral fitting and confirm several key results of previous surveys. Age gradients tend to be shallow for both early-type and late-type galaxies. As well known from previous studies, metallicity gradients are often complex (and not well modeled by linear or loglinear functions of radius), varying in detail from galaxy to galaxy on small radial scales. On average, however, over radial scales of order $1 R_{\mathrm{e}}$, MaNGA data provide the strongest statistical constraints to date that metallicity gradients are negative in both early- and late-type galaxies and are significantly steeper in disks. These results continue to indicate that the radial dependence of chemical enrichment processes is far more pronounced in disks than in spheroids, and indeed the relatively flat gradients in early-type galaxies are inconsistent with monolithic collapse. For both early- and late-type galaxies, more massive galaxies have steeper negative metallicity and age gradients. Since early-type galaxies tend to be more luminous, the overall steeper age and metallicity gradients in late-type galaxies reflect the fact that the trends in 
these gradients with galaxy mass are more pronounced for latetype galaxies. Goddard et al. (2017a) take advantage of the unique MaNGA sample size and mass range to characterize this correlation between metallicity and age gradients and galaxy mass, which explains the scatter in gradient values seen in previous studies.

Ibarra-Medel et al. (2016) meanwhile infer spatially resolved stellar mass assembly histories for the MaNGA galaxies, extending previously known relations between galaxy type and assembly history to a larger mass range. Their findings are consistent with blue/star-forming/late-type galaxies assembling, on average, from inside to out. Red/quiescent/early-type galaxies present a more uniform spatial mass assembly, or at least one that has been dynamically well mixed since star formation ceased, consistent with the flatter gradients seen, e.g., by Goddard et al. (2017a). In general, low-mass galaxies show evidence of more irregular global and spatial assembly histories than massive galaxies.

In a developing effort to model stellar population gradients, Johnston et al. (2017) demonstrate a new technique using MaNGA data that seeks to decompose the underlying population into contributions from different physical subcomponents. They explore how the disk and bulge components in galaxies reached their current states with a new approach to fit the 2D light profiles of galaxies as a function of wavelength and to isolate the spectral properties of these galaxies' disks and bulges. The MaNGA data have a spatial sampling of $0 . " 5$ per pixel, and successful decompositions were carried out with galaxies observed with the 61- to 127-fiber IFUs with fields of view of $22^{\prime \prime}$ to $32^{\prime \prime}$ in diameter, respectively.

S. B. Rembold et al. (2017, in preparation) have identified a "control sample" to the MaNGA luminous AGN host galaxies, matched on mass, distance, morphology, and inclination. Their conclusions, based on SDSS-III spectra of the central region, can be tested via evaluation of the stellar populations throughout the galaxies with MaNGA data.

\subsubsection{Gas and Stellar Kinematics}

Several studies are investigating the kinematics of both stars and gas across the galaxy population. Li et al. (2017) perform dynamical modeling on a more extensive and diverse sample of elliptical and spiral galaxies than had previously been done. By comparing the stellar mass-to-light ratios estimated from stellar population synthesis and from dynamics, they find a similar systematic variation of the initial mass function (IMF) to previous investigations. Early-type galaxies (elliptical and lenticular) with lower stellar mass-to-light ratios or velocity dispersions within one effective radius are consistent with a Chabrier-like IMF, while galaxies with higher stellar mass-tolight ratios or velocity dispersions are consistent with a more bottom-heavy IMF such as the Salpeter IMF.

Two studies have taken advantage of the large MaNGA sample in DR13 to quantify the frequency and attributes of galaxies with strong disparities between gas and stellar kinematics. Chen et al. (2016) find that an appreciable fraction of galaxies have counter-rotating gas and stars. Counterrotation is found in about $2 \%$ of all blue galaxies. The central regions of blue counter-rotators show younger stellar populations and more intense star formation than in their outer parts. Jin et al. (2016) have further studied the properties of 66 galaxies with kinematically misaligned gas and stars. They find that the star-forming misaligned galaxies have positive gradients in $D_{n} 4000$ and higher gas-phase metallicity, while the green valley/quiescent ones have negative $D_{n} 4000$ gradients and lower gas-phase metallicity on average. Despite this distinction, there is evidence that all types of kinematically misaligned galaxies tend to be in more isolated environments. They propose that misaligned star-forming galaxies originate from gas-rich progenitors accreting external kinematic decoupled gas, while the misaligned green valley/quiescent galaxies might be formed from accreting kinematic decoupled gas from dwarf satellites.

Finally, Penny et al. (2016) examine the kinematics of a sample of 39 quenched low-mass galaxies. The majority $(87 \%)$ of these quenched low-mass galaxies exhibit coherent rotation in their stellar kinematics, and a number host distinct disks or spiral features. Just five (13\%) are found to have rotation speeds $v_{\text {circ }}<15 \mathrm{~km} \mathrm{~s}^{-1}$ at $1 R_{e}$ and are dominated by pressure support at all radii. Two of the quenched low-mass galaxies (5\%) host kinematically distinct cores, with the stellar population at their centers counter-rotating with respect to the rest of the galaxy. The results support a picture in which the majority of quenched low-mass $(d E)$ galaxies have a disk origin.

\section{SEQUELS: eBOSS, TDSS, and SPIDERS Data}

DR13 includes the data from 126 plates observed under the SEQUELS program. This program was started in SDSS-III as an ancillary program to take advantage of some of the dark time released when BOSS was completed early. The SEQUELS targets were quite different from BOSS targets because the program was designed to finalize the eBOSS target selection algorithms. The primary targets were defined by two different SDSS-WISE selection algorithms to determine the eBOSS LRG program (Prakash et al. 2016) and several optical-IR and variability selections to determine the eBOSS quasar program (Myers et al. 2015). Likewise, objects selected from a combination of X-ray and optical imaging were used to determine the final SPIDERS cluster (Clerc et al. 2016) and AGN (Dwelly et al. 2017) programs, while variability in PanSTARRS imaging was used to determine the final TDSS program (Morganson et al. 2015; Ruan et al. 2016).

A total of 66 SEQUELS plates were completed in the final year of SDSS-III. The remaining 60 plates required to fill out the $400 \mathrm{deg}^{2}$ footprint were completed in the first year of SDSS-IV. As mentioned above, these data served a crucial role for verification of the eBOSS, TDSS, and SPIDERS target samples. SEQUELS and eBOSS LRG spectra were used to optimize the performance of a new redshift classification scheme that now reliably classifies more than $90 \%$ of eBOSS LRG spectra (Hutchinson et al. 2016), thus meeting the ambitious goal set forth at the beginning of the program (Dawson et al. 2016). The sample also seeds the eBOSS footprint to be used for clustering studies. The first clustering measurements from SEQUELS and eBOSS LRGs were just released (Zhai et al. 2017), and first results from quasar clustering are expected in the near future. All SEQUELS targets are tracked by the EBOSS_TARGETO bitmask. The Appendix of the DR12 paper (Alam et al. 2015) provides the motivation and description of each target selection algorithm captured by that bitmask. 


\section{1. eBOSS in SEQUELS}

A total of 117 plates from SEQUELS used a slightly broader selection for LRGs, clustering quasars $(0.9<z<2.2)$, and Ly $\alpha$ forest quasars to ensure that the final eBOSS selection would be included in each of these classes. Because the eBOSS sample is included in this region, the SEQUELS spectroscopy obtained in SDSS-III and SDSS-IV will be used directly in any LRG or quasar clustering studies. Nine plates from SEQUELS, all released in DR12, included targets derived from an early test of the ELG selection algorithm (Alam et al. 2015). These tests of ELG selection algorithms were part of a larger series of tests performed during SDSS-III and SDSS-IV (Comparat et al. 2016; Raichoor et al. 2016; Delubac et al. 2017. The spectra from these tests were also used in one of the first science results from eBOSS, a study of galactic-scale outflows traced by UV emission (hu et al. 2015). The selection algorithm used in these fields is quite different from what will be used in eBOSS, and these targets will not contribute to the final clustering sample. For this reason, we summarize the findings of the LRG and quasar spectra below but reserve discussion of the ELG spectra for future work.

DR13 does contain value-added catalogs relevant to the ELG sample. Raichoor et al. (2016) describe the Fischer discriminant used to select ELG targets using photometry from SDSS, WISE, and SCUSS (Zou et al. 2016) for the main ELG sample. Delubac et al. (2017) produce the catalogs used for ELG targeting with SDSS+WISE or SDSS +WISE+SCUSS data. Finally, Zhu et al. (2015) generated composite, continuumnormalized spectra of emission-line galaxies in the rest-UV from ELGs observed by BOSS in various programs. Table 2 lists the location of these files on the SAS.

\subsubsection{Luminous Red Galaxies from WISE Colors}

The increase in redshift complicates selection both by shifting the $4000 \AA$ break into the $i$-band filter and by requiring fainter targets than those observed in BOSS. WISE $3.4 \mu \mathrm{m}$ photometry (W1 band) was introduced to enable selection of this higher-redshift sample. As part of the SEQUELS program, two overlapping selections for LRGs at higher redshifts $(0.6<z<1.0$, vs. $0.4<z<0.7$ for CMASS $)$ were employed, allowing tests of potential strategies for eBOSS. Color cuts that combine optical and infrared photometry were employed, enabling the targeting of LRGs at these redshifts while maintaining a high purity. This selection technique takes advantage of the strong peak at a rest-frame wavelength of 1.6 $\mu \mathrm{m}$ that is present in the spectrum of most galaxies. This peak enters $\mathrm{W} 1$ as the redshift gets closer to 1, yielding large differences between the optical/IR colors of $z>0.6$ galaxies and stars.

SEQUELS selected a total of $\sim 70,000$ LRGs over an area of $\sim 700 \mathrm{deg}^{2}$ with $120.0<$ R.A. $<210.0$ and $45.0<$ decl. $<60.0$. These LRGs were selected by algorithms utilizing two different optical-IR color spaces, utilizing either SDSS $r, i, z$, and W1 or only $i, z$, and $\mathrm{W} 1$; the selection efficiency and redshift success for each algorithm could then be compared. The parameters of the selection algorithms were tuned such that each yielded a target density of $\sim 60 \mathrm{deg}^{-2}$; the two selections overlap significantly, yielding a net density of 72 targets $\mathrm{deg}^{-2}$. Figure 10 of Prakash et al. (2016) presents the resulting redshift distributions from each selection. The $r / i / z /$ WISE selection has been chosen for eBOSS, due to greater efficiency at selecting high-redshift LRGs. More details on the SEQUELS LRG sample selection can be found in Prakash et al. (2016).

\subsubsection{Quasars Targeted with Optical+WISE Photometry and Photometric Variability}

SEQUELS observations helped define a uniform quasar sample for eBOSS clustering studies based on ugriz and WISE photometry. The "Extreme Deconvolution" (XDQSO; Bovy et al. 2011a, 2011b) selection is used to identify likely quasars at redshifts $z>0.9$ according to the relative density of stars and quasars as a function of color, magnitude, and photometric uncertainty. The selection alone results in a highly complete sample of quasars to be used for clustering studies, but with contamination from stars that is too large to fit into the eBOSS fiber budget. The XDQSO selection is supplemented by morphology cuts to remove low-redshift AGNs and opticalIR colors to remove stellar objects with blackbody spectra. Variability data from the Palomar Transient Factory (PTF; Law et al. 2009) are used to supplement the selection of Ly $\alpha$ forest quasars, producing tracers with a density of $3.2 \mathrm{deg}^{-2}$ where sufficient PTF imaging data are available. In addition to cosmological measurements, the quasar sample can be used to study quasar astrophysics and galaxy evolution through studies of the quasar luminosity function, composite spectra, and multiwavelength spectral energy distributions spanning from the radio to the X-ray. Myers et al. (2015) found that $\sim 96 \%$ of all quasar targets with $r<22$ will be confidently classified. Section 5 of Myers et al. (2015) provides information on the properties of quasars observed with SEQUELS, including the numbers observed in the redshift ranges $0.9<z<2.1$ and $z>2.1$.

DR13 includes value-added catalogs with the variability measurements (see Palanque-Delabrouille et al. 2011) using either PTF data (Myers et al. 2015) or Stripe 82 data (PalanqueDelabrouille et al. 2016) that are used for selecting quasars in eBOSS based on variability. The locations of the FITS tables are given in Table 2.

\subsubsection{Redmonster and Improved Redshifts for LRGs}

The DR13 redshifts for all SEQUELS targets are determined in the usual fashion, with best-fitting combinations of PCA eigenspectra. Hutchinson et al. (2016) describe a new pipeline, redmonster, that uses a suite of discrete theoretical galaxy spectra as a basis to determine the most likely redshift through a minimum $\chi^{2}$, rather than linear combinations of templates used in Bolton et al. (2012). This pipeline achieves a 90.5\% automated redshift and spectral classification success rate for the LRG target class, a significant improvement over the performance of the previous pipeline. A value-added catalog using the new redmonster algorithms for the LRG sample is included in DR13. All spectra identified by EBOSS_TARGETO bit 1 or 2 were classified by redmonster. The file is named redmonsterAll-v5_9_0. fits and is found on the SAS as described in Table 2.

\subsection{SPIDERS in SEQUELS}

The main goal of the SPIDERS survey is to characterize a subset of X-ray sources identified by eROSITA using optical spectra from the BOSS spectrograph. The extended sources will mostly be galaxy clusters, which can be used for cosmology. The point sources will mainly be AGNs, which 


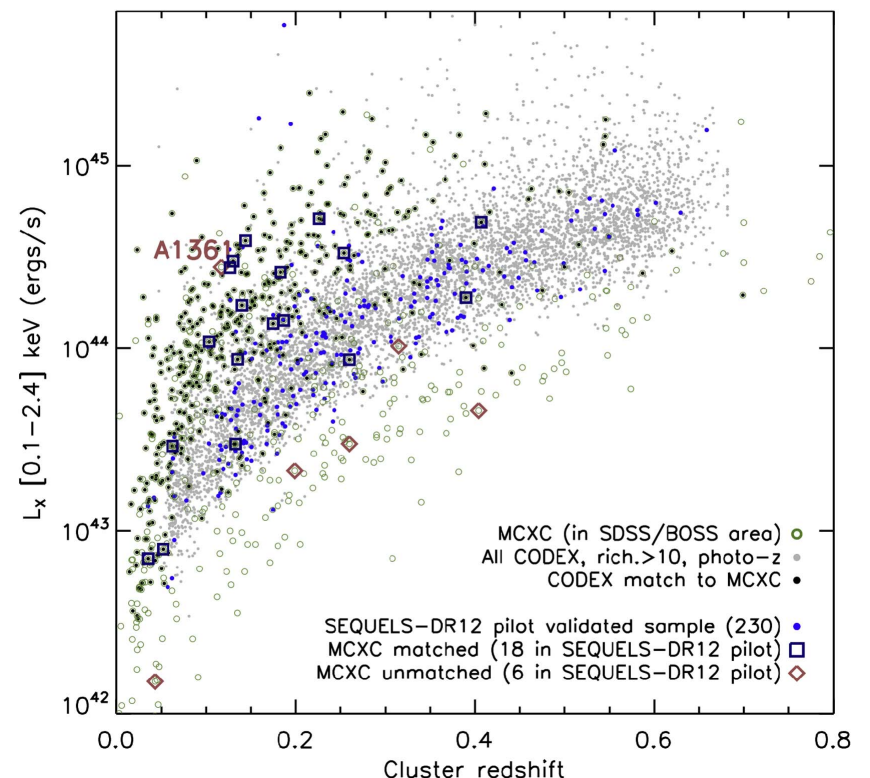

Figure 7. Distribution in the X-ray luminosity-redshift plane of galaxy clusters, adapted from Clerc et al. (2016). The gray circles mark the location of all candidate CODEX clusters using their photometric redshift. They form the main pool of cluster targets in SPIDERS. The CODEX candidate clusters that have already been spectroscopically confirmed and included in the MCXC meta-catalog (Piffaretti et al. 2011) are indicated by open symbols. The confirmed clusters lie mainly at higher X-ray luminosities. The 230 confirmed clusters in the SPIDERS DR13 value-added catalog (blue circles) extend the redshift range of known clusters in the SDSS area to $z \sim 0.6$ in a systematic way. Among these 230 , only 18 match clusters from the MCXC meta-catalog. The new clusters in general lie at lower X-ray luminosities and therefore probe lower X-ray masses than previous ROSATbased analyses. The six clusters indicated by red diamonds are MCXC clusters in the SEQUELS-DR12 footprint not present in SPIDERS (note that ABELL 1361 is within a masked area of the CODEX survey, so it is not matched).

can be used to study the evolution of black holes across cosmic time. For the first phase of SDSS-IV, when eROSITA data are not yet available, SPIDERS will be targeting based on ROSAT and $X M M$ data. The target catalogs for galaxy clusters and AGNs for SPIDERS from these two satellites have been included in DR13 as value-added catalogs. The SPIDERS AGN target catalogs (Dwelly et al. 2017) contain 9,028 candidate targets from RASS and 819 from XMMSL (XMMNewton Slew survey catalog; Saxton et al. 2008). They enclose information on the X-ray sources, including flux measurements, and a quantitative measure of the reliability of the association to optical and AllWISE data. The SPIDERS Galaxy Cluster target list (Clerc et al. 2016) contains 94,883 and 3,839 objects for RASS and XMM, respectively.

In SEQUELS, SPIDERS used similar targeting catalogs, also available as value-added products, to test targeting strategies and provide initial results. The selection criteria are somewhat different from the final SPIDERS algorithms. Full details are available in Clerc et al. (2016) and Dwelly et al. (2017). We summarize the SEQUELS SPIDERS data available in DR13 below.

\subsubsection{Optical Spectra of Galaxies in X-Ray-identified Clusters}

The cluster pilot study (Clerc et al. 2016) takes advantage of the CODEX (Constrain Dark Energy with X-ray clusters; A. Finoguenov et al. 2017, in preparation) candidate cluster list,

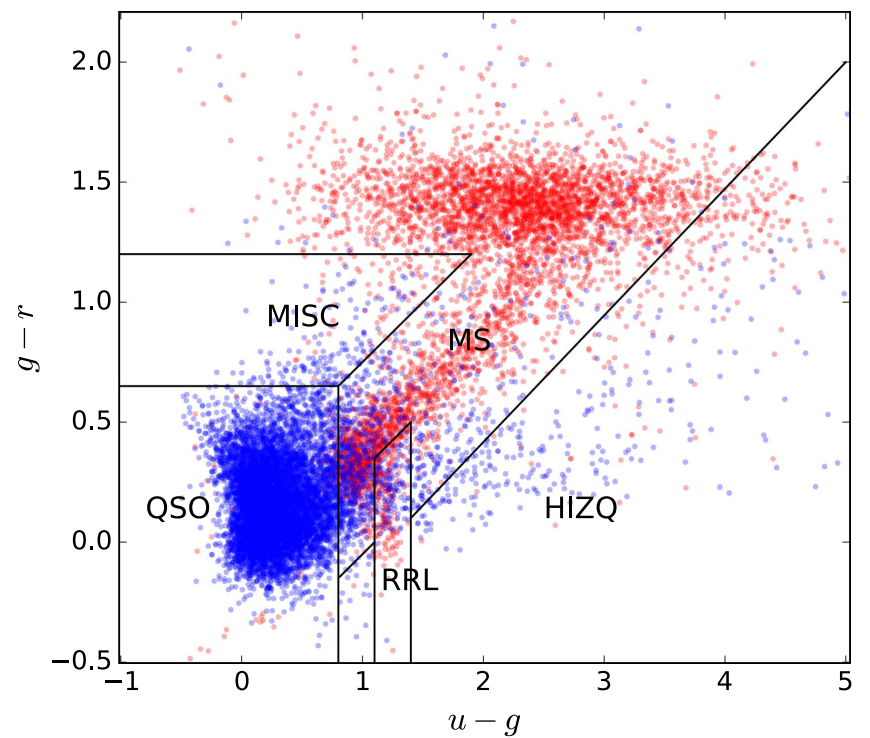

Figure 8. Representation of the TDSS spectroscopic characterization of imaging variables selected (from PanSTARRS 1 and SDSS imaging) without any primary bias based on color or specific light-curve character. Nearly 16,000 TDSS photometric variables in the region of the sky covered by the 66 initial SEQUELS plates are plotted here in a traditional color-color diagram. Their classifications are based on spectra taken in SDSS-I to SDSS-III, including SEQUELS data. Regions in color space traditionally considered as occupied by quasars, main-sequence stars, RR Lyrae, high-redshift quasars, and other miscellaneous subclasses are overlaid (see Sesar et al. 2007, but here using specific boundaries detailed in Morganson et al. 2015). The blue symbols depict TDSS photometric variables whose SDSS spectra verify them as quasars, while the red symbols depict TDSS variables whose SDSS spectra verify them as stars. A few hundred objects that were identified as galaxies or that could not be identified are not included in this plot. The wide distribution, as well as the overlap (in some regimes relatively densely), of both star and quasar symbols verifies that TDSS variability selection finds not only traditional quasars but also those within color regimes commonly attributed to stars (and with analogous results for the spectroscopically confirmed variable stars).

which is based on currently available RASS data. As part of DR13, we provide the catalog of X-ray-detected galaxy clusters spectroscopically confirmed using SEQUELS-DR12 SPIDERS spectroscopic data. Galaxy clusters were identified through the emission of their hot baryonic component as extended X-ray sources in RASS. The optical counterparts were found by optimally searching photometric data for the existence of a red sequence formed by their member galaxies. Spectroscopic redshifts obtained by SPIDERS provide definitive confirmation of the clustered nature of these objects and their redshift (up to $z \sim 0.6$ ). We assigned cluster membership combining an algorithm and visual validation of individual objects. The gas properties derived from X-ray observations (luminosity, temperature, $R_{200}{ }^{134}$ ) are derived using precise cluster redshifts $\left(\Delta_{z} \sim 0.001\right)$. We compute galaxy cluster velocity dispersions using several methods adapted to the low number of spectroscopic members per system (of the order 15-40), and we show that their values correlate with cluster X-ray luminosities, within expectations. Figure 7 shows the distribution of clusters with SEQUELS redshifts and membership in the redshift-Xray luminosity plane.

\footnotetext{
${ }^{134} R_{200}$ is defined as usual as the radius where the mean overdensity is equal to $200 \times$ the critical density.
} 


\subsubsection{Optical Spectra of X-Ray-identified AGNs}

The addition of X-ray-identified AGNs to the suite of AGNs with well-sampled redshifts helps complete the inventory of AGNs and trace black hole growth throughout cosmic history. SDSS has been observing optically identified AGNs since its inception under the main large-scale structure surveys, special targeting programs, and as "mistakes" in other targeting classes. In addition, there were several BOSS ancillary programs focused on X-ray follow-up, including a highly complete program on the XMM-XXL north field (Menzel et al. 2016). The SPIDERS SEQUELS program has added spectra of 274 ROSAT AGNs to the SDSS sample, identified on the basis of their SDSS colors only. The DR12 paper (Alam et al. 2015) and Dwelly et al. (2017) provide details on the targeting of these AGNs.

\subsection{TDSS in SEQUELS}

Nearly 18,000 targets selected or co-selected by TDSS have been observed among the 126 SEQUELS spectroscopic plates. The targeting strategy for TDSS in SEQUELS was very similar to that ultimately chosen for the bulk of SDSS-IV (Morganson et al. 2015). Ruan et al. (2016) present TDSS spectroscopic results from the 66 initial DR12 SEQUELS plates, along with a description of the small differences in targeting within SEQUELS. Figure 8 depicts results for the initial TDSS SEQUELS subsample. The classification of spectra was initially done using the BOSS pipeline (Bolton et al. 2012), but the spectra were also visually inspected. Overall, the pipeline performance is outstanding, with the highest-level spectral classification (e.g., star versus galaxy versus quasar) in agreement with our visual inspection for about $97 \%$ of the TDSS spectra and with only $2 \%$ of the pipeline redshifts for quasars requiring significant adjustment.

About $90 \%$ of the TDSS spectroscopic fibers are aimed at initial classification spectra for variables chosen without primary bias based on color or specific light-curve character. Their variability is determined from within PS1 multi-epoch imaging (Kaiser et al. 2010), or via longer-term photometric variability between SDSS and PS1 imaging surveys (Morganson et al. 2015). The initial SEQUELS results reveal comparable numbers of stars and quasars among these photometric variables. A summary for TDSS quasars is that the PS1/SDSS variability criteria mitigate some of the (wellknown) redshift biases of color selection, yielding a smooth and broad quasar redshift distribution, and that TDSS selects relatively redder quasars (e.g., than the eBOSS core quasar sample), as well as a higher fraction of some peculiar AGNs (such as BALQSOs and BL Lacs); and among variable stars, TDSS selects significant numbers of active late-type stars, stellar pulsators such as RR Lyrae, and eclipsing and composite binaries, along with smaller subsets of white dwarfs, cataclysmic variables, and carbon stars (see Ruan et al. 2016).

The other $\sim 10 \%$ of the TDSS targets are objects already having one or more earlier epochs of SDSS-I to SDSS-III spectroscopy, and for which TDSS is taking a second (or sometimes third, fourth, etc.) epoch to reveal anticipated spectroscopic variability. These "Few Epoch Spectroscopy" (or FES) targets include various subsets of quasars and stars (Morganson et al. 2015). Recent example science papers representative of this FES category of TDSS include work on acceleration of broad absorption lines in BALQSOs (e.g., Grier et al. 2016) and recent discoveries adding to the rare class of "changing look quasars" (e.g., Runnoe et al. 2016).

\subsection{Retrieving SEQUELS Data}

All SDSS data releases are cumulative, and therefore the SEQUELS data, whether taken in SDSS-III or SDSS-IV, have been reduced with the latest pipelines and included with all previous SDSS optical spectra data in this data release. SEQUELS targets can be identified because the EBOSS_TARGET0 bit will be set. The summary spAll-v5_9_0.fits data file, which includes classification information from the pipeline, is at https://data.sdss.org/sas/dr13/eboss/spectro/redux/v5_9_ $0 /$ on the SAS or in the specObjAll table on the CAS.

\section{APOGEE-2: Improved Spectral Extraction and Spectroscopic Parameters for APOGEE-1 Data}

The data released in DR13 are based on the same raw data as in DR12, and the pipelines for reduction and analysis remain similar to those used in DR13. First, the data reduction pipeline (Nidever et al. 2015) reduced the 3D raw data cubes into wellsampled, combined, sky-subtracted, telluric-corrected, and wavelength-calibrated 1D spectra. The stellar parameters and abundances were derived using ASPCAP (APOGEE Stellar Parameters and Chemical Abundances Pipeline; García Pérez et al. 2016) by finding the $\chi^{2}$ minimum when comparing the normalized observed spectra against a grid of synthetic spectra. However, the processing and analysis have been improved in several ways:

1. The line list used for determining stellar parameters and abundances has been revised.

2. Abundances are derived for several more species ( $\mathrm{C}$ I, P, Ti II, $\mathrm{Co}, \mathrm{Cu}, \mathrm{Ge}, \mathrm{Rb}$ ) than in DR12.

3. Results are now available for stars with $T_{\text {eff }}<3500$ using a newly employed MARCS model atmosphere grid.

4. Separate synthetic spectral grids are used for dwarfs and giants; results for dwarfs include rotation, and different isotope ratios are used for dwarfs and giants.

5. An initial, approximate attempt has been made to account for variable LSFs as a function of fiber number.

6. The correction for telluric absorption has been improved, primarily from a better LSF characterization, leading to better recovery of the stellar spectra.

7. The relation adopted from microturbulence has been refined, and a relation for macroturbulence has been incorporated.

More details are provided in J. Holtzman et al. (2017, in preparation; H16). Overall, these changes have improved and enhanced the APOGEE stellar parameters and abundances, but users need to continue to be aware of potential issues and data flagging, as discussed on the SDSS web site and in H16. In particular, parameters and abundances for low-metallicity stars and for cool stars are more uncertain.

Subsequent to the freezing of the DR13 release, several issues were discovered with the "calibrated" effective temperatures and surface gravities that are released with DR13. Based on good agreement of the spectroscopic effective temperature with photometric effective temperatures for the bulk of the sample that is near solar metallicity, no calibration was applied to the DR13 spectroscopic effective temperatures. It now appears, however, that these are systematically offset 
from photometric temperatures for stars of lower metallicity by as much as $200-300 \mathrm{~K}$ for stars at $[\mathrm{Fe} / \mathrm{H}]^{135} \sim-2$. For surface gravities, a calibration was derived based on asteroseismic surface gravities. While this calibration is good for most of the sample, it now appears that it does not yield accurate surface gravities for metal-poor stars; the calibrated surface gravities for stars with $[\mathrm{M} / \mathrm{H}]^{136}<-1.5$ are systematically too low, by as much as $\sim 0.5$ dex. H16 and the SDSS web site discuss both of these issues and suggest post-release calibrations.

\subsection{Improvements in Line List and Data Analysis}

Several improvements were made with regard to the APOGEE LSF. The characterization of the LSF was improved in one of the detectors, and an initial attempt was made at accommodating the LSF variability in the parameter and abundance pipeline by constructing spectral libraries for four different LSFs and using the most appropriate one for the analysis of each star.

The line list adopted for DR13, linelist.20150714, is an updated version of the one used for DR12 results (Shetrone et al. 2015) and is available at http://data.sdss.org/sas/dr13/ apogee/spectro/redux/speclib/linelists/. Shetrone et al. (2015) noted a number of concerns with the DR12 line list, which have been corrected in the new version (H16). As in DR12, the molecular line list is a compilation of literature sources including transitions of $\mathrm{CO}, \mathrm{OH}, \mathrm{CN}, \mathrm{C} 2, \mathrm{H} 2$, and $\mathrm{SiH}$. The $\mathrm{CN}$ line list has been updated from the DR12 version using a compilation from C. Sneden (2017, private communication). All molecular data are adopted without change, with the exception of a few obvious typographical corrections. The atomic line list was compiled using a number of literature sources for the lower excitation state of the transition and the transition probability, which is usually characterized as the " $g f$ " or " $\log (g f)$ " value. Literature sources include theoretical, astrophysical, and laboratory $g f$ values. A few new lines were added from NIST $5^{137}$ and other literature publications since the DR12 line list was created, including hyperfine splitting components for $\mathrm{Al}$ and $\mathrm{Co}$. We still rely heavily on "astrophysical" $g f$ values for atomic lines, where the transition probabilities of individual lines are determined using the spectrum of a star with known parameters and abundances, to construct line lists in the $H$ band (Meléndez \& Barbuy 1999; Ryde \& Schultheis 2015). For our line list, we use the centerof-disk spectrum of the Sun (Livingston \& Wallace 1991) and the full-disk spectrum of the nearby, well-studied, red giant Arcturus (Hinkle et al. 1995). To calculate the astrophysical $g f$ values, we used Turbospectrum (Alvarez \& Plez 1998; Plez 2012) to generate synthetic spectra of the center of disk for the Sun and the integrated disk for Arcturus with varying oscillator strengths and damping values to fit the solar and Arcturus spectra. In DR12, a different stellar atmosphere code was used for the $g f$ determination, and the synthetic grid creation and synthetic integrated disk spectra were used for the both the Sun and Arcturus. Although the change in $g f$ values from these changes is small (Shetrone et al. 2015), the DR13 line list is more self-consistently generated than previous versions. In DR12, we derived final astrophysical $g f$ values by

\footnotetext{
${ }^{135}[\mathrm{X} / \mathrm{H}]=\left(\log _{10}\left(N_{\mathrm{X}} / N_{\mathrm{H}}\right)\right)_{*}-\left(\log _{10}\left(N_{\mathrm{X}} / N_{\mathrm{H}}\right)\right)_{\odot} . \quad[\mathrm{X} / \mathrm{Fe}]=[\mathrm{X} / \mathrm{H}]_{*}-$ $[\mathrm{Fe} / \mathrm{H}]_{*}$.

${ }^{136}[\mathrm{M} / \mathrm{H}]$ is closely related to $[\mathrm{Fe} / \mathrm{H}]$. See Mészáros et al. (2013) for more details.

137 http://www.nist.gov/pml/data/asd.cfm
}

weighting the solar astrophysical $g f$ values at twice those of Arcturus. The astrophysical $g f$ solutions in DR13 are weighted according to line depth in Arcturus and in the Sun, to give more weight to where the relatively weak lines in the $H$ band produce the best signal, which usually gives more weight to the Arcturus solution. For lines with laboratory oscillator strengths, the astrophysical $\log (g f)$ values were not allowed to vary beyond twice the error quoted by the source.

\subsection{Additional Elements}

In DR13, APOGEE provides abundances for elements $\mathrm{P}, \mathrm{Cr}$, $\mathrm{Co}, \mathrm{Cu}, \mathrm{Ge}$, and $\mathrm{Rb}$ for the first time. The abundances of $\mathrm{C}, \mathrm{N}$, $\mathrm{O}, \mathrm{Na}, \mathrm{Mg}, \mathrm{Al}, \mathrm{Si}, \mathrm{S}, \mathrm{Ca}, \mathrm{Sc}, \mathrm{Ti}, \mathrm{V}, \mathrm{Mn}, \mathrm{Fe}$, and $\mathrm{Ni}$ were rederived. We added two new species: $\mathrm{CI}$ and Ti II, which provide valuable checks on the abundances derived from $\mathrm{CH}$ and Ti I lines (H16). The technique for calculating abundances of individual elements is described in Holtzman et al. (2015). As for DR12 and as discussed in Holtzman et al. (2015), we correct abundance ratios $[\mathrm{X} / \mathrm{Fe}]$, except for $[\mathrm{C} / \mathrm{Fe}]$ and $[\mathrm{N} /$ $\mathrm{Fe}]$, so there is no trend with temperature among the members of a single star cluster. In Figure 9, we show box plots for the abundances for member stars in four clusters with a range of metallicity. Both the mean values and the rms illustrate key points about the APOGEE DR13 abundances:

1. The elements cover a wide range in nucleosynthesis sites and atomic number. APOGEE is measuring the abundances of $\alpha$-elements, odd- $Z$ elements, iron-peak elements, and neutron-capture elements, as well as the mass, mixing, and AGB-contribution diagnostics $\mathrm{C}$ and $\mathrm{N}$.

2. The high $[\mathrm{X} / \mathrm{Fe}]$ for the $\alpha$-elements in the metal-poor $([\mathrm{Fe} / \mathrm{H}]<-0.7)$ globular clusters is present as expected (Wallerstein 1962). The increased scatter in $\mathrm{O}, \mathrm{Mg}$, and $\mathrm{Al}$ for these same clusters is in part due to the well-known light-element anomalies in globular clusters (e.g., Kraft 1994; Mészáros et al. 2015). The lower [Mn/Fe] in metal-poor stars is consistent with previous work (e.g., Wallerstein 1962; Gratton 1989) using 1D models that assume local thermodynamic equilibrium.

3. The increased scatter at lower metallicities is not entirely the result of actual inhomogeneities in cluster members, as there are increasing uncertainties associated with the weaker lines in more metal-poor stars. The error estimates reported in DR13 for these stars track the measured rms reasonably well, especially for errors in $[\mathrm{X} / \mathrm{Fe}]>0.1$ dex, and therefore provide a useful guide for abundance ratios that are not well determined (H16). On top of this, the ASPCAP methodology breaks down at some level for stars in which abundances within a given "abundance group" (e.g., the $\alpha$-elements) depart from solar abundance ratios, as is the case for second-generation stars in metal-poor globular clusters (Mészáros et al. 2015).

4. ASPCAP reports the parameters of the best-fitting model in $\chi^{2}$ space. It does not currently report nondetections and upper limits. Therefore, most reported abundances for elements such as $\mathrm{P}$ that show $\sim 1$ dex scatter in Figure 9 are based on fits to noise in the spectrum and do not reflect actual abundances. Abundances for such elements should not be used unless they are confirmed by visual inspection. They are reported because for a certain range in metallicity and temperature the lines can be detected 

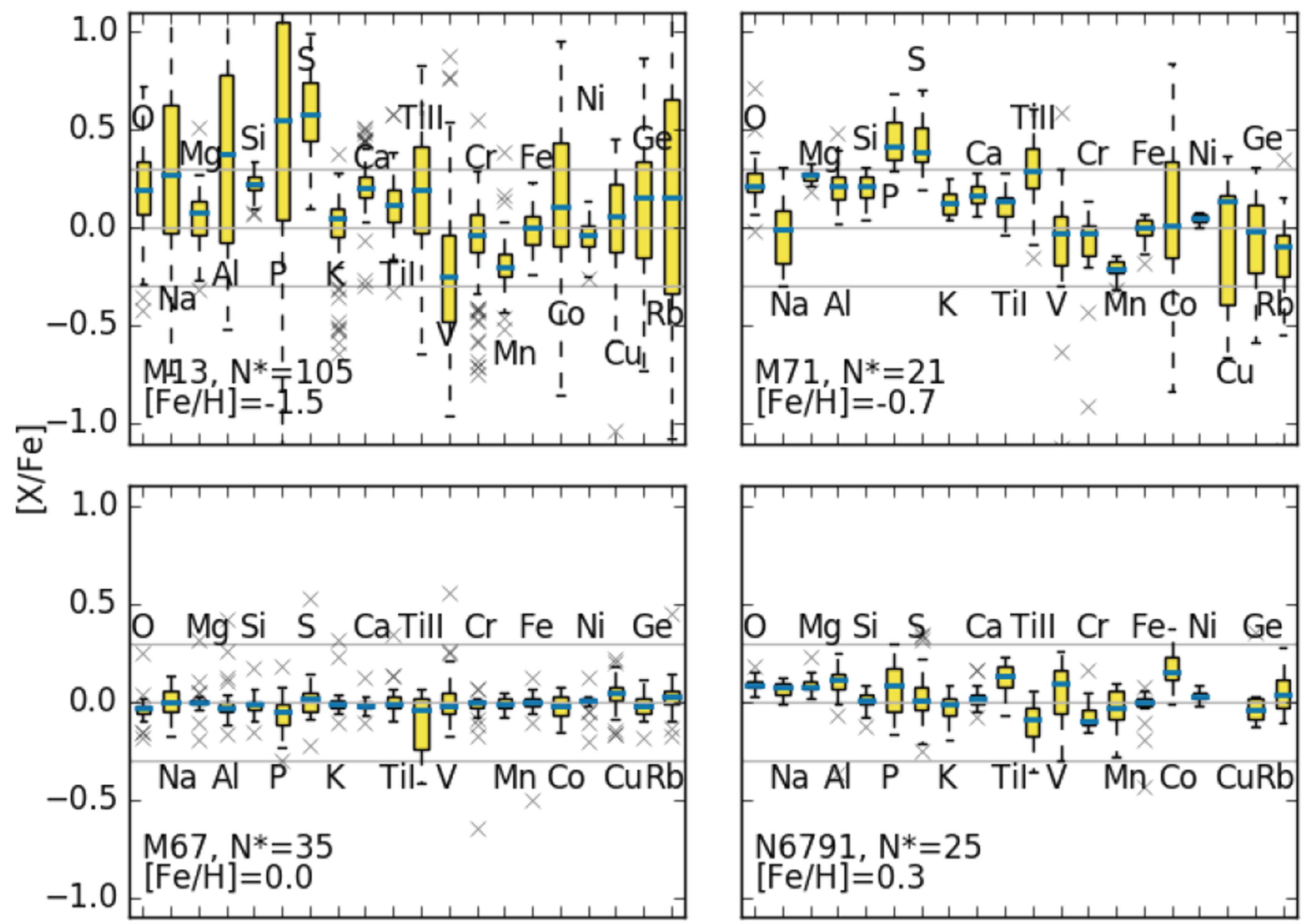

\section{Elements}

Figure 9. Box plot of the stars with $4100 \mathrm{~K}<T_{\text {eff }}<5000 \mathrm{~K}$ in four clusters spanning a range in metallicities $(\mathrm{M} 13=-1.58, \mathrm{M} 71=-0.82, \mathrm{M} 67=0.06$, NGC $6791=0.37$; Holtzman et al. 2015). $\mathrm{C}$ and $\mathrm{N}$ are not included because their values in a cluster change depending on the evolutionary state of the star as the result of first dredge-up (e.g., Masseron \& Gilmore 2015). A boxed horizontal line indicates the interquartile range and median found for a particular element. The vertical tails extending from the boxes indicate the total range of abundances determined for each element, excluding outliers. Outliers are shown by crosses.

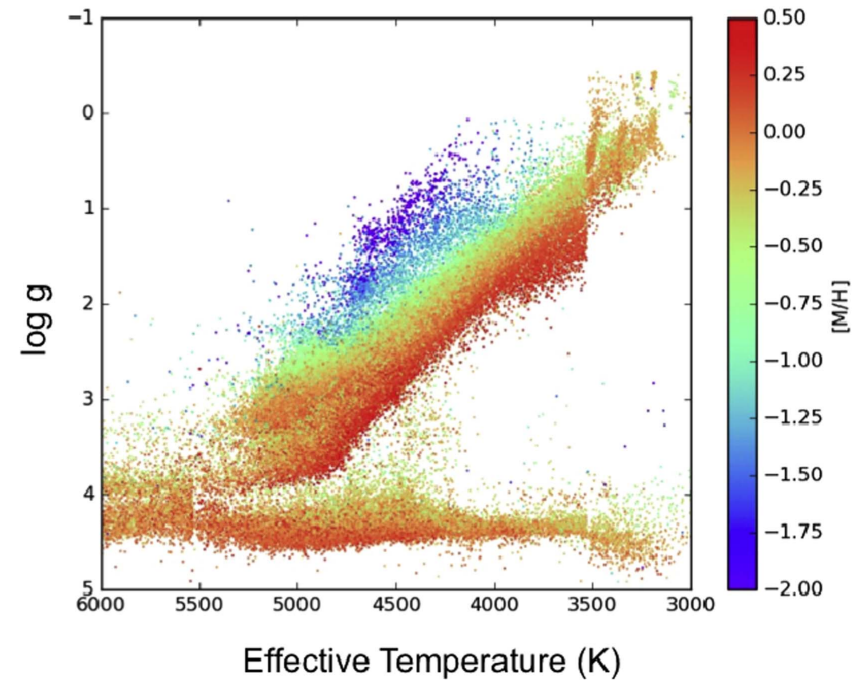

Figure 10. Hertzsprung-Russell diagram for all DR13 APOGEE-1 stars. The calibrated gravities and temperatures are used. The cool stars now clearly fall into dwarf and giant categories, but the stellar parameters do not merge smoothly onto the warmer Kurucz-based grid. and interesting chemistry exposed (e.g., Hawkins et al. 2016).

5 . The disagreement between the $[\mathrm{Ti} / \mathrm{Fe}]$ value based on Ti I lines and Ti II lines is yet to be resolved. We are currently investigating the choice of lines (as noted by Hawkins et al. 2016) and the effect of 3D and NLTE corrections.

6. The mean and scatter shown in Figure 9 were calculated using stars with $4100 \mathrm{~K}<T_{\text {eff }}<5000 \mathrm{~K}$. The scatter becomes noticeably worse if the warmest or coolest stars in the clusters are included. Warmer stars have weaker lines in general in the $H$ band, and the coolest stars are affected by the issues with the cool grid (see below).

The elements included in DR13 are by no means the only elements/species with lines present in the $H$ band amenable to abundance analysis. Hawkins et al. (2016), for example, independently derive $\mathrm{Yb}$, along with many other elements, for the APOKASC sample (Pinsonneault et al. 2014). We expect to include additional elements in upcoming data releases. 


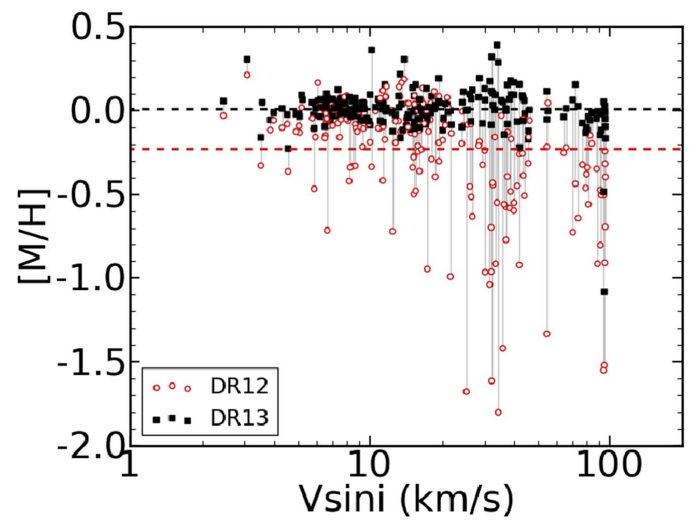

Figure 11. Comparison of the $[\mathrm{M} / \mathrm{H}]$ derived for Pleiades cluster dwarfs from DR12 (red open circles) and DR13 (black filled squares). The Pleiades has many rapidly spinning stars with rotationally broadened, shallow lines. When analyzed with the DR12 version of ASPCAP, which did not include rotational broadening, the best $\chi^{2}$ fit for the high $v \sin i$ stars was achieved for synthetic spectra with metallicity much lower than that known for the Pleiades. Therefore, the cluster average was subsolar (red line). In DR13, where the dwarfs are run through a grid that includes a $v \sin i$ dimension, all Pleiades stars fall close to the correct value (black line), regardless of broadening.

\subsection{Synthetic Spectral Grids at $T_{\text {eff }}<3500 \mathrm{~K}$ and with Rotational Broadening}

In DR10-12, the synthetic spectral grid used by ASPCAP (Mészáros et al. 2012; Zamora et al. 2015) was restricted to temperatures hotter than $3500 \mathrm{~K}$ because Kurucz model atmospheres are not available at cooler temperatures. However, many important APOGEE targets have cooler temperatures, including the luminous metal-rich $\mathrm{M}$ giants in the bulge, cool asymptotic giant branch stars, and M-dwarf planet hosts. Therefore, we used custom MARCS (Gustafsson et al. 2008) atmospheres provided by B. Edvardsson (2017, private communication) to construct new synthetic spectra. For the stellar atmospheres for the giants, the atmospheres are spherical; otherwise, they are plane-parallel. The Kurucz model grid and the new MARCS model grid overlap between 3500 and $4000 \mathrm{~K}$. In this region, there are some systematic differences between the results from the two grids; to provide homogeneous results, DR13 adopts the parameters and abundances from the analysis with the Kurucz grid.

Figure 10 shows the current parameter space in $T_{\text {eff }}, \log g$, and $[\mathrm{M} / \mathrm{H}]$ covered by Data Release 13 stars. It is immediately apparent that the parameters derived from the MARCS grid do not match smoothly to the parameters from the Kurucz grid. Possible explanations include the switch from plane-parallel Kurucz to spherical MARCS for the giants and/or the large number of model atmospheres in the MARCS grid that failed to converge. Because ASPCAP requires a square grid of synthetic spectra, these "grid holes" were filled by adjacent model atmospheres. The line list does not have FeH lines, which can be important features in the atmospheres of cool M dwarfs. We are investigating the size of the error caused by using incorrect model atmospheres in the grid, the possibilities of using alternative methods of interpolating and identifying the best-fit model, and the addition of FeH lines. H16 provides more details on the construction of the cool grid and the resulting stellar parameters. Nonetheless, Figure 10 illustrates the improved parameter space over DR12, which should aid in classifying $\mathrm{M}$ stars correctly as dwarfs or giants and separate the early $\mathrm{M}$ from the late $\mathrm{M}$ stars.
The APOGEE-1 sample is dominated by giants used to probe the chemical cartography of the Galaxy (Majewski et al. 2017). Fewer than $2 \%$ of red giants rotate at speeds detectable at the APOGEE spectrograph resolution (de Medeiros et al. 1996; Carlberg et al. 2011). Therefore, the first versions of ASPCAP did not include rotational broadening as a dimension in the synthetic spectral grid, which substantially reduced the computing time. However, dwarf stars, of which there are $>20,000$ in APOGEE-1, are frequently rapidly rotating, especially if they are young. For DR13, we added a dimension to the synthetic spectral grid where spectra were broadened by a Gaussian kernel. To keep the computing time reasonable, and in acknowledgment of the small effect that $\mathrm{C}$ and $\mathrm{N}$ abundances have on the atmospheres of warm dwarfs, we fixed the $[\mathrm{C} / \mathrm{M}]$ and $[\mathrm{N} / \mathrm{M}]$ grids to solar values. The $[\mathrm{C} / \mathrm{Fe}]$ and $[\mathrm{N} / \mathrm{Fe}]$ values reported for dwarfs in DR13 are calculated from windows after the best-fit parameters are determined, in similar fashion to the other individual elements. Figure 11 shows the improvement in the stellar parameters for members of the Pleiades star cluster. Some of the dwarfs in this young cluster are rotating with $v \sin i>50 \mathrm{~km} \mathrm{~s}^{-1}$. In DR12, ASPCAP found a best-fit solution for metal-poor stars. The shallower the lines because of rotational broadening, the more metal-poor the star was reported to be. With the DR13 grid, there is no longer a prominent trend in $[\mathrm{M} / \mathrm{H}]$ with $v \sin i$, and the mean value of the cluster is now at the expected metallicity.

\subsection{Data Access}

Data access via the CAS and SAS is similar to that for DR12 (Holtzman et al. 2015); "dr12" should be replaced with "dr13" in the path name or DR13 as the context in CASJobs. Some of the tag/column names have been modified. While raw abundances are still given in the FELEM arrays, "calibrated" abundances are now presented in X_H and X_M arrays, as well as in individual X_FE tags/columns. For dwarfs, there is now a column in the allStar fits file on the SAS or in the aspcapStar table on the CAS that reports the $v \sin i$. DR13 also includes a new catalog of red-clump stars based on the new ASPCAP parameters, following the method of Bovy et al. (2014), available at the location described in Table 2.

\section{The Future}

SDSS-IV will continue to add to the SDSS legacy of data and data analysis tools in upcoming data releases. DR14 is scheduled for 2017 July 31, and there will be yearly data releases until the end of the survey in 2020. For MaNGA, future data releases will include more data cubes of galaxies that are currently being observed. DR14 will include 2744 additional galaxies observed by MaNGA. In DR15, a data interface system that includes web- and python-based tools to access a data analysis pipeline that carries out continuum and emission-line fitting to provide estimates of stellar and gas kinematics, emission-line fluxes, and absorption-line index measurements will be released. In addition, MaNGA has started a bright-time observing program, piggybacking on APOGEE-2, to build a stellar library. These reduced optical stellar spectra will be included in DR15. For eBOSS, future data releases will provide sufficient redshifts of LRGs, ELGs, and quasars to be of cosmological interest, on its way to the goals described in Section 1. For example, DR14 contains the spectra for $2480 \mathrm{deg}^{2}$ observed by eBOSS. TDSS and 
SPIDERS will also release many more spectra, redshifts, and classifications for variable and X-ray-emitting objects, respectively. For APOGEE-2, the next data release (DR14) will contain spectra for an additional 98,882 stars from the APOGEE-2N spectrograph at APO observed under SDSS-IV, and DR16 and subsequent DRs will provide spectra taken with both the APOGEE-2N and APOGEE-2S spectrographs at LCO. In addition to observing red giants as the main target category, data will be obtained for RR Lyrae stars in the bulge, dwarf spheroidal galaxies, the Magellanic Clouds, Kepler Objects of Interest, and targets in the K2 fields.

We would like to thank the Center for Data Science (http:/ cds.nyu.edu) at New York University for their hospitality during DocuFeest 2016.

Funding for the Sloan Digital Sky Survey IV has been provided by the Alfred P. Sloan Foundation, the U.S. Department of Energy Office of Science, and the Participating Institutions. SDSS-IV acknowledges support and resources from the Center for High-Performance Computing at the University of Utah. The SDSS web site is http://www.sdss.org.

SDSS-IV is managed by the Astrophysical Research Consortium for the Participating Institutions of the SDSS Collaboration, including the Brazilian Participation Group, the Carnegie Institution for Science, Carnegie Mellon University, the Chilean Participation Group, the French Participation Group, HarvardSmithsonian Center for Astrophysics, Instituto de Astrofísica de Canarias, Johns Hopkins University, Kavli Institute for the Physics and Mathematics of the Universe (IPMU)/University of Tokyo, Lawrence Berkeley National Laboratory, Leibniz Institut für Astrophysik Potsdam (AIP), Max-Planck-Institut für Astronomie (MPIA Heidelberg), Max-Planck-Institut für Astrophysik (MPA Garching), Max-Planck-Institut für Extraterrestrische Physik (MPE), National Astronomical Observatory of China, New Mexico State University, New York University, University of Notre Dame, Observatário Nacional/MCTI, The Ohio State University, Pennsylvania State University, Shanghai Astronomical Observatory, United Kingdom Participation Group, Universidad Nacional Autónoma de México, University of Arizona, University of Colorado Boulder, University of Oxford, University of Portsmouth, University of Utah, University of Virginia, University of Washington, University of Wisconsin, Vanderbilt University, and Yale University.

\section{ORCID iDs}

Brett H. Andrews (iD https://orcid.org/0000-0001-8085-5890 Alfonso Aragón-Salamanca (iD https://orcid.org/0000-00018215-1256

Vladimir Avila-Reese (ib https://orcid.org/0000-00023461-2342

Carles Badenes (ib https://orcid.org/0000-0003-3494-343X

Kat Barger (1) https://orcid.org/0000-0001-5817-0932

Jorge Barrera-Ballesteros (D) https://orcid.org/0000-00032405-7258

Sarbani Basu (iD https://orcid.org/0000-0002-6163-3472

Timothy C. Beers (i) https://orcid.org/0000-0003-4573-6233

Matthew Bershady (iD https://orcid.org/0000-0002-3131-4374

Dmitry Bizyaev (i) https://orcid.org/0000-0002-3601-133X

Michael Blanton (D) https://orcid.org/0000-0003-1641-6222

Jo Bovy (i) https://orcid.org/0000-0001-6855-442X
Joel R. Brownstein (1D https://orcid.org/0000-0002-8725-1069

Kevin Bundy (iD https://orcid.org/0000-0001-9742-3138

Michele Cappellari (i) https://orcid.org/0000-0002-1283-8420

Ricardo Carrera (ib https://orcid.org/0000-0001-6143-8151

Edmond Cheung (1) https://orcid.org/0000-0001-8546-1428

Drew Chojnowski iD https://orcid.org/0000-0001-9984-0891

Haeun Chung (iD https://orcid.org/0000-0002-3043-2555

Kevin Covey (iD https://orcid.org/0000-0001-6914-7797

Jeffrey D. Crane (i) https://orcid.org/0000-0002-5226-787X

Jeremy Darling (iD https://orcid.org/0000-0003-2511-2060

Kyle Dawson (iD https://orcid.org/0000-0002-0553-3805

Nathan De Lee (i) https://orcid.org/0000-0002-3657-0705

Niv Drory (iD https://orcid.org/0000-0002-7339-3170

Arthur Eigenbrot (iD https://orcid.org/0000-0003-0810-4368

Eric Emsellem (1) https://orcid.org/0000-0002-6155-7166

Michael Eracleous (10) https://orcid.org/0000-0002-3719-940X

Xiaohui Fan (DD https://orcid.org/0000-0003-3310-0131

Diane Feuillet (i) https://orcid.org/0000-0002-3101-5921

Scott W. Fleming (i) https://orcid.org/0000-0003-0556-027X

Peter Frinchaboy (iD https://orcid.org/0000-0002-0740-8346

Hai Fu (iD https://orcid.org/0000-0001-9608-6395

Yang Gao (高扬) (iD https://orcid.org/0000-0002-6316-1632

Ana E. Garcia Pérez (10 https://orcid.org/0000-0003-

2184-6198

Patrick Gaulme (1) https://orcid.org/0000-0001-8330-5464

Douglas Geisler (i) https://orcid.org/0000-0002-3900-8208

Léo Girardi (iD https://orcid.org/0000-0002-6301-3269

Paul Green (ib https://orcid.org/0000-0002-8179-9445

Catherine J. Grier (i) https://orcid.org/0000-0001-9920-6057

Hong Guo (iD https://orcid.org/0000-0003-4936-8247

Alex Hagen (ib https://orcid.org/0000-0003-2031-7737

Paul Harding (iD https://orcid.org/0000-0003-3442-6248

Suzanne Hawley (iD https://orcid.org/0000-0002-6629-4182

Saskia Hekker (i) https://orcid.org/0000-0002-1463-726X

David W. Hogg (1) https://orcid.org/0000-0003-2866-9403

Kelly Holley-Bockelmann (iD https://orcid.org/0000-00032227-1322

Jon A. Holtzman (iD https://orcid.org/0000-0002-9771-9622

Jian Hu (胡剑) (10 https://orcid.org/0000-0002-2439-2438

Daniel Huber (i) https://orcid.org/0000-0003-4894-9779

Timothy Alan Hutchinson (10) https://orcid.org/0000-0002-

3009-260X

Ho Seong Hwang (iD https://orcid.org/0000-0003-3428-7612

Trey W. Jensen (i) https://orcid.org/0000-0003-2777-5116

Jennifer A. Johnson (iD https://orcid.org/0000-0001-7258-1834

Eric Jullo (ib https://orcid.org/0000-0002-9253-053X

T. Kallinger (iD https://orcid.org/0000-0003-3627-2561

Karen Kinemuchi (iD https://orcid.org/0000-0001-7908-7724

Juna A. Kollmeier (iD https://orcid.org/0000-0001-9852-1610

Ivan Lacerna (ib https://orcid.org/0000-0002-7802-7356

Dustin Lang (i) https://orcid.org/0000-0002-1172-0754

David R. Law (i) https://orcid.org/0000-0002-9402-186X

Alexie Leauthaud (iD https://orcid.org/0000-0002-3677-3617

Cheng Li (1) https://orcid.org/0000-0002-8711-8970

Lihwai Lin

(林俐暉) (ib https://orcid.org/0000-0001-7218-7407

Lin Lin (林琳) (10 https://orcid.org/0000-0003-1138-8146

Yen-Ting Lin

(林彥廷) (10 https://orcid.org/0000-0001-7146-4687

Sara Lucatello (i) https://orcid.org/0000-0001-8808-0073

Suvrath Mahadevan (ib https://orcid.org/0000-0001-9596-7983 
Steven R. Majewski iㅏ https://orcid.org/0000-00032025-3147

Arturo Manchado (1) https://orcid.org/0000-0002-3011-686X Rui Marques-Chaves (10 https://orcid.org/0000-00018442-1846

Karen L. Masters (i) https://orcid.org/0000-0003-0846-9578 Ian D. McGreer (1) https://orcid.org/0000-0002-3461-5228 Michael R. Merrifield (ib https://orcid.org/0000-00024202-4727

Andres Meza (i) https://orcid.org/0000-0002-9460-7828

Andrea Miglio (1) https://orcid.org/0000-0001-5998-8533

Ivan Minchev (i) https://orcid.org/0000-0002-5627-0355

Kirpal Nandra (1) https://orcid.org/0000-0002-7150-9192

Jeffrey A. Newman (10) https://orcid.org/0000-0001-8684-2222

David L. Nidever (1) https://orcid.org/0000-0002-1793-3689

Julia O'Connell (ㄷ) https://orcid.org/0000-0003-2321-950X

Nelson Padilla (1) https://orcid.org/0000-0001-9850-9419

Kaike Pan (1) https://orcid.org/0000-0002-2835-2556

Ismael Perez-Fournon (1) https://orcid.org/0000-00022807-6459

Marc H. Pinsonneault (i https://orcid.org/0000-00027549-7766

Abhishek Prakash (i) https://orcid.org/0000-0003-4451-4444

Mubdi Rahman (1) https://orcid.org/0000-0003-1842-6096

Hans-Walter Rix (1) https://orcid.org/0000-0003-4996-9069

Carlos Román-Zúñiga (i) https://orcid.org/0000-0001-

8600-4798

John Ruan (1) https://orcid.org/0000-0001-8665-5523

Mara Salvato (i) https://orcid.org/0000-0001-7116-9303

Sebastian F. Sanchez $\mathbb{1}$ https://orcid.org/0000-0001-

6444-9307

Jaderson S. Schimoia $\odot$ https://orcid.org/0000-0002-

5640-6697

Eddie Schlafly @i https://orcid.org/0000-0002-3569-7421

David J. Schlegel (1) https://orcid.org/0000-0002-5042-5088

Ralph Schönrich (10) https://orcid.org/0000-0002-4236-3091

Mathias Schultheis (1) https://orcid.org/0000-0002-6590-1657

Branimir Sesar (1) https://orcid.org/0000-0002-0834-3978

Matthew Shetrone (1) https://orcid.org/0000-0003-0509-2656

Michael Shull (1) https://orcid.org/0000-0002-4594-9936

Victor Silva Aguirre (1) https://orcid.org/0000-0002-

6137-903X

David V. Stark i https://orcid.org/0000-0002-3746-2853

Keivan G. Stassun (1) https://orcid.org/0000-0002-3481-9052

Matthias Steinmetz (1) https://orcid.org/0000-0001-6516-7459

Dennis Stello (ㄷ) https://orcid.org/0000-0002-4879-3519

Michael A. Strauss (1) https://orcid.org/0000-0002-0106-7755

Guy S. Stringfellow iㅏ https://orcid.org/0000-0003-1479-3059

Genaro Suarez (ํ) https://orcid.org/0000-0002-2011-4924

Baitian Tang (i) https://orcid.org/0000-0002-0066-0346

Jamie Tayar (10) https://orcid.org/0000-0002-4818-7885

Nicholas Troup (10) https://orcid.org/0000-0003-3248-3097

Jonathan R. Trump (i) https://orcid.org/0000-0002-1410-0470

Remco Van den Bosch (1) https://orcid.org/0000-0002-

0420-6159

David Wake (1) https://orcid.org/0000-0002-6047-1010

Rene Walterbos (10) https://orcid.org/0000-0002-0782-3064

Enci Wang (1) https://orcid.org/0000-0003-1588-9394

David H. Weinberg (1) https://orcid.org/0000-0001-7775-7261

W. M. Wood-Vasey (i) https://orcid.org/0000-0001-7113-1233

Dominika Wylezalek 다 https://orcid.org/0000-0003-

2212-6045
Renbin Yan (1) https://orcid.org/0000-0003-1025-1711

Jason E. Ybarra (1) https://orcid.org/0000-0002-3576-4508

Nadia Zakamska (1) https://orcid.org/0000-0001-6100-6869

Kai Zhang 나 https://orcid.org/0000-0002-9808-3646

Gong-Bo Zhao (i) https://orcid.org/0000-0003-4726-6714

Zheng Zheng (i) https://orcid.org/0000-0003-1887-6732

Zhi-Min Zhou (i) https://orcid.org/0000-0002-4135-0977

Guangtun Zhu (1) https://orcid.org/0000-0002-7574-8078

Joel C. Zinn (1) https://orcid.org/0000-0002-7550-7151

Hu Zou (i) https://orcid.org/0000-0002-6684-3997

\section{References}

Abazajian, K. N., Adelman-McCarthy, J. K., Agüeros, M. A., et al. 2009, ApJS, 182, 543

Alam, S., Albareti, F. D., Allende Prieto, C., et al. 2015, ApJS, 219, 12

Alam, S., Ata, M., Bailey, S., et al. 2017, MNRAS, 470, 2617

Alatalo, K., Blitz, L., Young, L. M., et al. 2011, ApJ, 735, 88

Allen, J. T., Croom, S. M., Konstantopoulos, I. S., et al. 2015, MNRAS, 446, 1567

Alvarez, R., \& Plez, B. 1998, A\&A, 330, 1109

Anguiano, B., De Silva, G. M., Freeman, K., et al. 2016, MNRAS, 457, 2078

Barrera-Ballesteros, J. K., Heckman, T. M., Zhu, G. B., et al. 2016, MNRAS, 463, 2513

Baumgartner, W. H., Tueller, J., Markwardt, C. B., et al. 2013, ApJS, 207, 19

Bautista, J. E., Busca, N. G., Guy, J., et al. 2017, A\&A, 603, A12

Belfiore, F., Maiolino, R., Bundy, K., et al. 2015, MNRAS, 449, 867

Belfiore, F., Maiolino, R., Maraston, C., et al. 2016, MNRAS, 461, 3111

Belfiore, F., Maiolino, R., Maraston, C., et al. 2017, MNRAS, 466, 2570

Beutler, F., Blake, C., Colless, M., et al. 2011, MNRAS, 416, 3017

Blanton, M. R., Bershady, M. A., Abolfathi, B., et al. 2017, AJ, 154, 28

Blanton, M. R., Kazin, E., Muna, D., Weaver, B. A., \& Price-Whelan, A. 2011, AJ, 142, 31

Blanton, M. R., Schlegel, D. J., Strauss, M. A., et al. 2005, AJ, 129, 2562

Bolton, A. S., Schlegel, D. J., Aubourg, É., et al. 2012, AJ, 144, 144

Bovy, J., Hennawi, J. F., Hogg, D. W., et al. 2011a, ApJ, 729, 141

Bovy, J., Nidever, D. L., Rix, H.-W., et al. 2014, ApJ, 790, 127

Bovy, Jo., Hogg, D. W., \& Roweis, S. T. 2011b, AnApS, 5, 1657

Bowen, I. S., \& Vaughan, A. H., Jr. 1973, ApOpt, 12, 1430

Brough, S., van de Sande, J., Owers, M. S., et al. 2017, ApJ, 844, 59

Bryant, J. J., Owers, M. S., Robotham, A. S. G., et al. 2015, MNRAS, 447,2857

Bundy, K., Bershady, M. A., Law, D. R., et al. 2015, ApJ, 798, 7

Cano-Díaz, M., Sánchez, S. F., Zibetti, S., et al. 2016, ApJL, 821, L26

Cappellari, M., Emsellem, E., Krajnović, D., et al. 2011, MNRAS, 413, 813

Carlberg, J. K., Majewski, S. R., Patterson, R. J., et al. 2011, ApJ, 732, 39

Chang, H.-Y., Song, Y.-H., Luo, A.-L., et al. 2017, ApJ, 834, 92

Chen, Y.-M., Shi, Y., Tremonti, C. A., et al. 2016, NatCo, 7, 13269

Cheung, E., Bundy, K., Cappellari, M., et al. 2016a, Natur, 533, 504

Cheung, E., Stark, D. V., Huang, S., et al. 2016b, ApJ, 832, 182

Clerc, N., Merloni, A., Zhang, Y.-Y., et al. 2016, MNRAS, 463, 4490

Cluver, M. E., Jarrett, T. H., Hopkins, A. M., et al. 2014, ApJ, 782, 90

Comparat, J., Delubac, T., Jouvel, S., et al. 2016, A\&A, 592, A121

Dalcanton, J. J., Williams, B. F., Seth, A. C., et al. 2009, ApJS, 183, 67

Davis, T. A., Alatalo, K., Sarzi, M., et al. 2011, MNRAS, 417, 882

Dawson, K. S., Kneib, J.-P., Percival, W. J., et al. 2016, AJ, 151, 44

Dawson, K. S., Schlegel, D. J., Ahn, C. P., et al. 2013, AJ, 145, 10

de Medeiros, J. R., Da Rocha, C., \& Mayor, M. 1996, A\&A, 314, 499

De Silva, G. M., Freeman, K. C., Bland-Hawthorn, J., et al. 2015, MNRAS, 449, 2604

Delubac, T., Bautista, J. E., Busca, N. G., et al. 2015, A\&A, 574, A59

Delubac, T., Raichoor, A., Comparat, J., et al. 2017, MNRAS, 465, 1831

Doi, M., Tanaka, M., Fukugita, M., et al. 2010, AJ, 139, 1628

Dong, S., Xie, J.-W., Zhou, J.-L., Zheng, Z., \& Luo, A. 2017, arXiv:1706. 07807

Dong, S., Zheng, Z., Zhu, Z., et al. 2014, ApJL, 789, L3

Drory, N., MacDonald, N., Bershady, M. A., et al. 2015, AJ, 149, 77

Dwelly, T., Salvato, M., Merloni, A., et al. 2017, MNRAS, 469, 1065

Eisenstein, D. J., Weinberg, D. H., Agol, E., et al. 2011, AJ, 142, 72

Eisenstein, D. J., Zehavi, I., Hogg, D. W., et al. 2005, ApJ, 633, 560

Fang, X.-S., Zhao, G., Zhao, J.-K., Chen, Y.-Q., \& Bharat Kumar, Y. 2016, MNRAS, 463, 2494 
Fernández-Trincado, J. G., Robin, A. C., Vieira, K., et al. 2015, A\&A, 583, A76

Finkbeiner, D. P., Schlafly, E. F., Schlegel, D. J., et al. 2016, ApJ, 822, 66 Fitzpatrick, E. L. 1999, PASP, 111, 63

Frasca, A., Molenda-Żakowicz, J., De Cat, P., et al. 2016, A\&A, 594, A39

Frieman, J. A., Bassett, B., Becker, A., et al. 2008, AJ, 135, 338

Fukugita, M., Ichikawa, T., Gunn, J. E., et al. 1996, AJ, 111, 1748

Gaia Collaboration, Brown, A. G. A., Vallenari, A., et al. 2016, A\&A, 595, A2

García Pérez, A. E., Allende Prieto, C., Holtzman, J. A., et al. 2016, AJ, 151,144

Geha, M., Blanton, M. R., Yan, R., \& Tinker, J. L. 2012, ApJ, 757, 85

Gilmore, G., Randich, S., Asplund, M., et al. 2012, Msngr, 147, 25

Goddard, D., Thomas, D., Maraston, C., et al. 2017a MNRAS, 465, 688

Goddard, D., Thomas, D., Maraston, C., et al. 2017b MNRAS, 466, 4731

Gomes, J. M., Papaderos, P., Kehrig, C., et al. 2016, A\&A, 588, A68

Gratton, R. G. 1989, A\&A, 208, 171

Green, A. W., Croom, S. M., Scott, N., et al. 2017, arXiv:1707.08402

Gunn, J. E., Carr, M., Rockosi, C., et al. 1998, AJ, 116, 3040

Gunn, J. E., Siegmund, W. A., Mannery, E. J., et al. 2006, AJ, 131, 2332

Guo, Q., White, S., Boylan-Kolchin, M., et al. 2011, MNRAS, 413, 101

Gustafsson, B., Edvardsson, B., Eriksson, K., et al. 2008, A\&A, 486, 951

Guzzo, L., Scodeggio, M., Garilli, B., et al. 2014, A\&A, 566, A108

Hawkins, K., Masseron, T., Jofré, P., et al. 2016, A\&A, 594, A43

Helmi, A., Veljanoski, J., Breddels, M. A., Tian, H., \& Sales, L. V. 2017, A\&A, 598, A58

Hinkle, K., Wallace, L., \& Livingston, W. 1995, PASP, 107, 1042

Hinton, S. R., Kazin, E., Davis, T. M., et al. 2017, MNRAS, 464, 4807

Ho, I.-T., Medling, A. M., Bland-Hawthorn, J., et al. 2016, MNRAS, 457, 1257

Holincheck, A. J., Wallin, J. F., Borne, K., et al. 2016, MNRAS, 459, 720

Holtzman, J. A., Shetrone, M., Johnson, J. A., et al. 2015, AJ, 150, 148

Hou, W., Luo, A., Yang, H., et al. 2015, MNRAS, 449, 1401

Howes, L. M., Asplund, M., Casey, A. R., et al. 2014, MNRAS, 445, 4241

Hutchinson, T. A., Bolton, A. S., Dawson, K. S., et al. 2016, AJ, 152, 205

Ibarra-Medel, H. J., Sánchez, S. F., Avila-Reese, V., et al. 2016, MNRAS, 463, 2799

Jacobson, H. R., Friel, E. D., Jílková, L., et al. 2016, A\&A, 591, A37

Jansen, F., Lumb, D., Altieri, B., et al. 2001, A\&A, 365, L1

Jarrett, T. H., Masci, F., Tsai, C. W., et al. 2013, AJ, 145, 6

Jin, Y., Chen, Y., Shi, Y., et al. 2016, MNRAS, 463, 913

Johnston, E. J., Häußler, B., Aragón-Salamanca, A., et al. 2017, MNRAS, 465, 2317

Kaiser, N., Aussel, H., Burke, B. E., et al. 2002, Proc. SPIE, 4836, 154

Kaiser, N., Burgett, W., Chambers, K., et al. 2010, Proc. SPIE, 7733, 77330E

Kazin, E. A., Koda, J., Blake, C., et al. 2014, MNRAS, 441, 3524

Kehrig, C., Monreal-Ibero, A., Papaderos, P., et al. 2012, A\&A, 540, A11

Kraft, R. P. 1994, PASP, 106, 553

Kunder, A., Bono, G., Piffl, T., et al. 2014, A\&A, 572, A30

Kunder, A., Kordopatis, G., Steinmetz, M., et al. 2017, AJ, 153, 75

Lang, D., Hogg, D. W., \& Schlegel, D. J. 2016, AJ, 151, 36

Law, D. R., Cherinka, B., Yan, R., et al. 2016, AJ, 152, 83

Law, D. R., Yan, R., Bershady, M. A., et al. 2015, AJ, 150, 19

Law, N. M., Kulkarni, S. R., Dekany, R. G., et al. 2009, PASP, 121, 1395

Li, C., Kauffmann, G., Jing, Y. P., et al. 2006, MNRAS, 368, 21

Li, C., Wang, E., Lin, L., et al. 2015, ApJ, 804, 125

Li, H., Ge, J., Mao, S., et al. 2017, ApJ, 838, 77

Li, H.-N., Zhao, G., Christlieb, N., et al. 2015, ApJ, 798, 110

Li, N., \& Thakar, A. R. 2008, CSE, 10, 18

Licquia, T. C., Newman, J. A., \& Brinchmann, J. 2015, ApJ, 809, 96

Livingston, W., \& Wallace, L. 1991, An Atlas of the Solar Spectrum in the Infrared from 1850 to $9000 \mathrm{~cm}^{-1}$ (1.1 to 5.4 micrometer) (Tucson, AZ: National Solar Observatory)

Magrini, L., Randich, S., Kordopatis, G., et al. 2017, A\&A, 603, A2

Majewski, S. R., Schiavon, R. P., Frinchaboy, P. M., et al. 2017, AJ, 154, 94

Martell, S. L., Sharma, S., Buder, S., et al. 2017, MNRAS, 465, 3203

Masseron, T., \& Gilmore, G. 2015, MNRAS, 453, 1855

Meléndez, J., \& Barbuy, B. 1999, ApJS, 124, 527

Menzel, M.-L., Merloni, A., Georgakakis, A., et al. 2016, MNRAS, 457, 110 Mészáros, S., Allende Prieto, C., Edvardsson, B., et al. 2012, AJ, 144, 120

Mészáros, S., Holtzman, J., García Pérez, A. E., et al. 2013, AJ, 146, 133

Mészáros, S., Martell, S. L., Shetrone, M., et al. 2015, AJ, 149, 153

Morganson, E., Green, P. J., Anderson, S. F., et al. 2015, ApJ, 806, 244

Mullaney, J. R., Alexander, D. M., Fine, S., et al. 2013, MNRAS, 433, 622

Myers, A. D., Palanque-Delabrouille, N., Prakash, A., et al. 2015, ApJS, 221, 27

Nidever, D. L., Holtzman, J. A., Allende Prieto, C., et al. 2015, AJ, 150, 173

Paegert, M., Stassun, K. G., De Lee, N., et al. 2015, AJ, 149, 186
Palanque-Delabrouille, N., Magneville, C., Yèche, C., et al. 2016, A\&A, 587, A41

Palanque-Delabrouille, N., Yèche, C., Borde, A., et al. 2013, A\&A, 559, A85

Palanque-Delabrouille, N., Yeche, C., Myers, A. D., et al. 2011, A\&A, 530, A122

Penny, S. J., Masters, K. L., Weijmans, A.-M., et al. 2016, MNRAS, 462, 3955

Pérez, E., Cid Fernandes, R., González Delgado, R. M., et al. 2013, ApJL, 764, L1

Piffaretti, R., Arnaud, M., Pratt, G. W., Pointecouteau, E., \& Melin, J.-B. 2011, A\&A, 534, A109

Piffl, T., Scannapieco, C., Binney, J., et al. 2014, A\&A, 562, A91

Pinsonneault, M. H., Elsworth, Y., Epstein, C., et al. 2014, ApJS, 215, 19

Plez, B. 2012, Turbospectrum, Astrophysics Source Code Library, ascl:1205.004

Prakash, A., Licquia, T. C., Newman, J. A., et al. 2016, ApJS, 224, 34

Predehl, P., Andritschke, R., Becker, W., et al. 2014, Proc. SPIE, 9144, 91441 T

Raichoor, A., Comparat, J., Delubac, T., et al. 2016, A\&A, 585, A50

Recio-Blanco, A., Rojas-Arriagada, A., de Laverny, P., et al. 2017, A\&A, 602, L14

Ren, A., Fu, J., De Cat, P., et al. 2016, ApJS, 225, 28

Robin, A. C., Bienaymé, O., Fernández-Trincado, J. G., \& Reylé, C. 2017, A\&A, 605, 1

Rojas-Arriagada, A., Recio-Blanco, A., de Laverny, P., et al. 2017, A\&A, 601, A 140

Ruan, J. J., Anderson, S. F., Green, P. J., et al. 2016, ApJ, 825, 137

Ruchti, G. R., Read, J. I., Feltzing, S., et al. 2015, MNRAS, 450, 2874

Runnoe, J. C., Cales, S., Ruan, J. J., et al. 2016, MNRAS, 455, 1691

Ryde, N., \& Schultheis, M. 2015, A\&A, 573, A14

Sánchez, S. F., García-Benito, R., Zibetti, S., et al. 2016, A\&A, 594, A36

Sánchez, S. F., Kennicutt, R. C., Gil de Paz, A., et al. 2012, A\&A, 538, A8

Sánchez, S. F., Rosales-Ortega, F. F., Iglesias-Páramo, J., et al. 2014, A\&A, 563, A49

Saxton, R. D., Read, A. M., Esquej, P., et al. 2008, A\&A, 480, 611

Schaefer, A. L., Croom, S. M., Allen, J. T., et al. 2017, MNRAS, 464, 121

Schlafly, E. F., \& Finkbeiner, D. P. 2011, ApJ, 737, 103

Schlafly, E. F., Finkbeiner, D. P., Jurić, M., et al. 2012, ApJ, 756, 158

Schlegel, D. J., Finkbeiner, D. P., \& Davis, M. 1998, ApJ, 500, 525

Sesar, B., Ivezić, Ž, Lupton, R. H., et al. 2007, AJ, 134, 2236

Shao, L., Kauffmann, G., Li, C., Wang, J., \& Heckman, T. M. 2013, MNRAS, 436, 3451

Shetrone, M., Bizyaev, D., Lawler, J. E., et al. 2015, ApJS, 221, 24

Shu, Y., Bolton, A. S., Schlegel, D. J., et al. 2012, AJ, 143, 90

Siebert, A., Famaey, B., Minchev, I., et al. 2011, MNRAS, 412, 2026

Smee, S. A., Gunn, J. E., Uomoto, A., et al. 2013, AJ, 146, 32

Smith, M. C., Ruchti, G. R., Helmi, A., et al. 2007, MNRAS, 379, 755

Steinmetz, M., Zwitter, T., Siebert, A., et al. 2006, AJ, 132, 1645

Thakar, A. R. 2008, CSE, 10, 9

Thakar, A. R., Szalay, A., Fekete, G., \& Gray, J. 2008, CSE, 10, 30

Thomas, N., Ge, J., Grieves, N., Li, R., \& Sithajan, S. 2016, PASP, 128, 045003

Ting, Y.-S., Conroy, C., \& Rix, H.-W. 2016, ApJ, 816, 10

Vasudevan, R. V., \& Fabian, A. C. 2009, MNRAS, 392, 1124

Voges, W., Aschenbach, B., Boller, T., et al. 1999, A\&A, 349, 389

Wake, D. A., Bundy, K., Diamond-Stanic, A. M., et al. 2017, AJ, 154, 86

Wallerstein, G. 1962, ApJS, 6, 407

Wilkinson, D. M., Maraston, C., Thomas, D., et al. 2015, MNRAS, 449, 328

Williams, M. E. K., Steinmetz, M., Binney, J., et al. 2013, MNRAS, 436, 101

Wright, E. L., Eisenhardt, P. R. M., Mainzer, A. K., et al. 2010, AJ, 140, 1868-81

Yan, R., Bundy, K., Law, D. R., et al. 2016, AJ, 152, 197

Yan, R., Tremonti, C., Bershady, M. A., et al. 2015, AJ, 151, 8

Yan, R., Tremonti, C., Bershady, M. A., et al. 2016, AJ, 151, 8

Yang, X., Mo, H. J., van den Bosch, F. C., et al. 2007, ApJ, 671, 153

Yanny, B., Rockosi, C., Newberg, H. J., et al. 2009, AJ, 137, 4377

York, D. G., Adelman, J., Anderson, J. E., Jr., et al. 2000, AJ, 120, 1579

Zamora, O., García-Hernández, D. A., Allende Prieto, C., et al. 2015, AJ, 149,181

Zasowski, G., Johnson, J. A., Frinchaboy, P. M., et al. 2013, AJ, 146, 81

Zhai, Z., Tinker, J. L., Hahn, C., et al. 2017, ApJ, 848, 76

Zhang, K., Yan, R., Bundy, K., et al. 2017, MNRAS, 466, 3217

Zhao, G., Zhao, Y.-H., Chu, Y.-Q., Jing, Y.-P., \& Deng, L.-C. 2012, RAA, 12,723

Zheng, Z., Wang, H., Ge, J., et al. 2017, MNRAS, 465, 4572

Zhu, G. B., Comparat, J., Kneib, J.-P., et al. 2015, ApJ, 815, 48

Zou, H., Zhou, X., Jiang, Z., et al. 2016, AJ, 151, 37 